\title{
Local entropy rigidity for hyperbolic manifolds
}

\author{
Livio Flaminio ${ }^{1}$
}

\begin{abstract}
We study deformations of compact hyperbolic manifolds of a given total volume. We show that along any non-trivial deformation the topological entropy and the difference between topological entropy and Liouville entropy are locally strictly convex functions of the deformation parameter, thus providing a partial positive answer to a conjecture of A. Katok.
\end{abstract}

\section{$\S 1$. Introduction.}

\subsection{Statement of the problem.}

1.1.1. Notation. Throughout this article, $M$ denotes a compact smooth manifold of dimension $n$. Given a $C^{2}$ Riemannian metric $g$ on $M$, the geodesic flow determined by $g$ on the unit tangent bundle $S_{g} M$ is denoted by $T_{g}$ or $T_{g}^{t}$, or simply by $g^{t}$ when this abuse of notation will not be confusing.

1.1.2. Geodesic flows on the unit tangent bundles $S_{g} M$ of compact Riemannian manifolds $(M, g)$ of negative curvature are the chief examples of transitive Anosov flows.

We recall that a continuous flow $T:(v, t) \in N \times \mathbb{R} \mapsto T^{t} v \in N$ is transitive if it has a dense orbit. A flow $T$ on a compact manifold $N$ is an Anosov flow [Ano67] if it is $C^{1}$ and the tangent bundle $T N$ of $N$ splits continuously in $T$-invariant subbundles $T N=E^{0} \oplus E^{u} \oplus E^{s}$ satisfying the conditions:

1. $E^{0}$ is the tangent space to the orbits of flow.

2. There exists positive constants $\lambda$ and $C$ such that $\left\|d T^{t} \mid E^{s}\right\|<C e^{-\lambda t}$ and $\left\|d T^{-t} \mid E^{u}\right\|<C e^{\lambda t}$ for all $t>0$.

\footnotetext{
${ }^{1}$ Work supported by NSF grant \# DMS-9101911 and MSRI
} 
1.1.3. Anosov flows have many invariant measures. Given a $T^{t}$-invariant probability measure $m$, we denote by $h(T, m)$ or $h\left(T^{t}, m\right)$ the measure theoretic entropy of the time 1 transformation $T^{1}$ with respect to the measure $m$ [Pet83]. The supremum

$$
h_{\mathrm{top}}(T)=\sup \left\{h(T, m) \mid T^{t} \text {-invariant probability measure } m\right\}
$$

of all measure theoretic entropies is attained, for Anosov flows, by a unique measure, the Margulis measure $m_{0}$, also called the measure of maximal entropy [Mar70, Bow72a, Bow74, BR75].

In the case of a geodesic flow $T_{g}^{t}$, we also have a smooth $T_{g}^{t}$-invariant measure on $S_{g} M$, the Liouville measure $\operatorname{Liouv}(g)$, which arises from the contact structure on $S_{g} M$.

1.1.4. For locally symmetric manifolds of negative curvature, it is not difficult to see that the Liouville measure and the Margulis measure coincide. In other words, for geodesic flows on locally symmetric manifolds of negative curvature, the measure theoretical entropy of Liouville measure coincides with the topological entropy. The converse of this statement is the content of the following conjecture of A. Katok.

1.1.5. Conjecture [Kat82, BK85]. The topological entropy and the entropy of the Liouville measure for a geodesic flow on a negatively curved manifold coincide, (if and) only if the manifold is locally symmetric.

A. Katok himself showed in [Kat82] that the conjecture is true if one considers metrics conformally equivalent to a locally symmetric metric. In particular, the conjecture is true for surfaces of genus greater than 1 .

The topological entropy has also another geometric interpretation [Man79]. Denote by $\widetilde{M}$ the universal cover of $M$. For a negatively curved metric $g$ on $M$, let $B_{g, r}(p)$ be the ball of radius $r$ centred at $p \in \widetilde{M}$ for the lift to $\widetilde{M}$ of metric $g$. Then

$$
h_{\mathrm{top}}\left(T_{g}\right)=\lim _{r \rightarrow \infty} \frac{1}{r} \log \operatorname{Vol}_{g}\left(B_{g, r}(p)\right) .
$$

In words, the topological entropy of the geodesic flow coincides with the volume growth of balls in the universal cover of $M$.

A related conjecture of Gromov, formulated at about the same time as Katok's conjecture, states: 
1.1.6. Conjecture ([Gro83]). Among all metrics of volume equal to the volume of a locally symmetric metric $g_{0}$ on a manifold $M$, the volume growth of balls in the universal cover $\widetilde{M}$ of $M$ is minimized at the metrics isometric to $g_{0}$.

Besson, Courtois and Gallot ([BCG94a] and [BCG94b]) have recently announced a proof of Gromov's conjecture for rank-one neg. curved locally symmetric manifolds which builds up on their 1992 theorem:

1.1.7. Theorem ([BCG]). Let $g_{0}$ be a metric of constant negative curvature on a compact manifold $M$ of dimension n. Then, in the space of $H^{s}$ metrics with volume equal to the volume of $g_{0}$, there exists a neighbourhood $\mathcal{U}$ of $g_{0}$ in the $H^{s}$ topology $(s>n / 2+2)$, such that the minimum of the topological entropy in $\mathcal{U}$ is attained only by metrics isometric to $g_{0}$.

1.1.8. In consideration of the fact that the method of Besson, Courtois and Gallot applies so far only to topological entropy, in order to shade some light on Katok's entropy conjecture 1.1.5, it is very interesting to study the functions

$$
\begin{gathered}
\text { Ent }_{\text {top }}: g \in \mathcal{M}^{r}(M) \mapsto h_{\text {top }}\left(T_{g}\right) \\
\text { Ent }_{\text {Liouv }}: g \in \mathcal{M}^{r}(M) \mapsto h\left(T_{g}, \operatorname{Liouv}(g)\right)
\end{gathered}
$$

in a neighbourhood of the locally symmetric metric $g_{0}$. Here we have denoted by $\left(M, g_{0}\right)$ a compact locally symmetric manifold of negative curvature and set

$$
\mathcal{M}^{r}(M)=\left\{C^{r} \text { metrics } g \text { on } M \mid \operatorname{Vol}_{g}(M)=\operatorname{Vol}_{g_{0}}(M)\right\} .
$$

1.1.9. We remark that the above functions Ent $t_{\text {top }}$ and Ent $t_{\text {Liouv }}$ are known to be smooth by previous works respectively of Katok, Knieper, Pollicott and Weiss [KKPW89] (for the topological entropy) and of Contreras (for the Liouville entropy) [Con92], provided we restrict ourselves to negatively curved metrics. It is also known [KKW91] that a locally symmetric metric $g_{0}$ is a critical point of Ent $t_{\text {top }}$ and Ent $t_{\text {Liouv }}$.

\subsection{Statement of the theorems.}

In this paper we obtain estimates for the second derivative of the functions Ent $t_{\text {top }}$ and Ent $_{\text {Liouv }}$ at a metric of constant negative curvature 
which allow us to establish that along a path through a metric of constant negative curvature $g_{0}$, locally, the only metric for which one has $\operatorname{Ent}_{\text {top }}(g)=\operatorname{Ent}_{\text {Liouv }}(g)$ is $g_{0}$. More exactly:

Theorem A. Let $g_{0}$ be a metric of constant negative curvature on a compact manifold $M$ of dimension $n$ and let $g_{\varepsilon}$ be a $C^{2}$ curve of $C^{5}$ metrics of constant volume. Then, if $g_{\varepsilon}$ is not tangent to the orbit of $g_{0}$ under the diffeomorphism group, the function

$$
\varepsilon \mapsto \operatorname{Ent}_{\text {top }}\left(g_{\varepsilon}\right)-\operatorname{Ent}_{\text {Liouv }}\left(g_{\varepsilon}\right)
$$

is strictly convex at $\varepsilon=0$. In particular, it follows that along the path $g_{\varepsilon}$ and for small $\epsilon$ 's, the equality $\operatorname{Ent}_{\text {top }}\left(g_{\varepsilon}\right)=\operatorname{Ent}_{\text {Liouv }}\left(g_{\varepsilon}\right)$ occurs only at $g_{0}$.

In [Pol94], Pollicott proved that at a locally symmetric metric the topological entropy is convex for volume preserving conformal deformations. Along the way to the proof of Theorem A we find the following theorem.

Theorem B. Under the same hypothesis as Theorem A, the function

$$
\varepsilon \mapsto \operatorname{Ent}_{\mathrm{top}}\left(g_{\varepsilon}\right),
$$

is locally strictly convex at $g_{0}$.

For the measure theoretical entropy the volume normalization does not yield any useful convexity or concavity property. Surprisingly, we have:

Theorem C. There exists an hyperbolic 3-manifold $\left(M, g_{0}\right)$ for which the function Ent Liouv $_{\text {: }} \mathcal{M}^{5}(M) \rightarrow R^{+}$has second derivative at $g_{0}$ with mixed signature. In particular the entropy Ent $_{\text {Liouv }}$ of the Liouville measure does not have either a minimum nor a maximum at $g_{0}$, when restricted to $\mathcal{M}^{5}(M)$.

\subsection{Outline of the proofs.}

The scheme for the estimate can be divided into three parts. In the first part, since geodesic flows on a negatively curved manifold can be represented as a flow built under a function on a topologically mixing subshift of finite type, we investigate these flows.

We recall some basic definitions about cocycles. Let $T$ be a Borel $\mathbb{R}$-flow on a Borel space $X$. An ( $\mathbb{R}$-valued) cocycle for the flow $T$ is a Borel function $c: X \times \mathbb{R} \rightarrow \mathbb{R}$ satisfying the relation

$$
c(x, t+s)=c(x, t)+c\left(T^{t} x, s\right) .
$$


Cocycles for the flow $T$ form a group under addition and a cocycle $c$ for $T$ is called a coboundary if $c(x, t)=b\left(T^{t} x\right)-b(x)$ for some Borel function $b$. Coboundaries form a subgroup and the elements of the quotient group are the Borel cohomology classes of the flow. If a cocycle $c$ is "differentiable along the orbits", i.e. if $c(x, t)=\int_{0}^{t} A\left(T^{s} x\right) d s$, for some Borel function $A$ on $X$, we say that $A$ generates $c$. In a cohomology class we can always find a representative which is smooth along the orbits. If $X$ has a $C^{\alpha}$ structure, we say that a cohomology class is $C^{\alpha}$ if it has a representative generated by a $C^{\alpha}$ function on $X$.

If $(X, T)$ is a symbolic flow, i.e. a flow built over a topologically mixing subshift of finite type with a Hölder ceiling function $\lambda$, then to each $C^{\alpha}$ cohomology class $[c]$ is attached a $T$-invariant measure $m_{[c]}$ called the Gibbs state for $[c]$. If $A$ is a Hölder function generating a representative of $[c]$, it is costumery to refer to $A$ as the potential for the Gibbs state $m_{[c]}$. The function identically equal to 1 , generates a cocycle, called the length cocycle and its corresponding Gibbs state is the measure of maximal entropy for $T$.

We prove the following Proposition about derivatives of entropy of Gibbs states for symbolic flows in terms of the variation of the generating cocycles. The $\operatorname{Cov}_{u}^{T}(v, w)$ below denotes the "covariance" of Hölder continuous functions $v, w$ for the flow $T$ and with respect to the Gibbs state for the potential $u$ (see [Rue78] and $\S 2$ ) and $\operatorname{Var}_{u}^{T}(v)=\operatorname{Cov}_{u}^{T}(v, v)$.

1.3.1. Proposition (A). Let $(\Sigma, \sigma)$ be a topologically mixing subshift of finite type and let $\lambda_{\varepsilon}$ be a $C^{2}$ curve positive $C^{\alpha}$ functions on $\Sigma$. Let $\left(X_{\varepsilon}, T_{\varepsilon}^{t}\right)$ be the special flow built over $(\Sigma, \sigma)$ with ceiling function $\lambda_{\varepsilon}$. Let $\delta l_{\varepsilon}$ be a $C^{\alpha}$ function on $X_{\varepsilon} \approx\left\{(p, t) \mid p \in \Sigma, 0 \leq t<\lambda_{\varepsilon}(p)\right\}$ with the property that

$$
\int_{0}^{\lambda_{\varepsilon}(p)} \delta l_{\varepsilon}(p, t) d t=d / d \varepsilon \lambda_{\varepsilon}(p) .
$$

Then, denoting with primes differentiations with respect to $\varepsilon$, we have :

$$
h_{\text {top }}^{\prime}\left(T_{0}\right) \equiv \frac{d}{d \varepsilon} h_{\text {top }}\left(T_{\varepsilon}\right)_{\varepsilon=0}=-h_{\text {top }}\left(T_{0}\right) m_{0}\left(\delta l_{0}\right)
$$

where $m_{0}$ is the measure of maximal entropy for the flow $T_{0}$. If $h_{\mathrm{top}}^{\prime}\left(T_{0}\right)=0$ and $\delta^{2} l_{\varepsilon}$ denotes a $C^{\alpha}$ function on $X_{\varepsilon}$ with the property that

$$
\int_{0}^{\lambda_{\varepsilon}(p)} \delta^{2} l_{\varepsilon}(p, t) d t=d^{2} / d \varepsilon^{2} \lambda_{\varepsilon}(p)
$$

then

$$
h_{\mathrm{top}}^{\prime \prime}\left(T_{0}\right)=-h_{\mathrm{top}}\left(T_{0}\right) M_{0}\left(\delta^{2} l_{0}\right)+h_{\mathrm{top}}\left(T_{0}\right)^{2} \operatorname{Var}_{0}^{T_{0}}\left(\delta l_{0}\right) .
$$


(B). Let $u_{\varepsilon}=u_{\varepsilon}(p, t),(p \in \Sigma, t \in \mathbb{R})$, be a $C^{2}$ family of Hölder continuous functions of pressure zero for the flow $T_{\varepsilon}$. Let $m_{u_{\varepsilon}}$ denote the Gibbs state for the flow $T_{\varepsilon}$ and potential $u_{\varepsilon}$. We have:

$$
h^{\prime}\left(T_{0}, m_{u_{0}}\right) \equiv \frac{d}{d \varepsilon} h\left(T_{\varepsilon}, m_{u_{\varepsilon}}\right)_{\varepsilon=0}=\operatorname{Cov}_{u_{0}}^{T_{0}}\left(u_{0}, \delta u_{0}\right)-h\left(T_{0}, m_{u_{0}}\right) m_{u_{0}}\left(\delta l_{0}\right),
$$

where $\delta u_{\varepsilon}$ is the (generator of) first variation of the cocycle generated by $u_{\varepsilon}$ and is defined by $\int_{0}^{\lambda_{\varepsilon}} \delta u_{\varepsilon}(p, t) d t=d / d \varepsilon \int_{0}^{\lambda_{\varepsilon}} u_{\varepsilon}(p, t) d t$. If $u_{0}$ is cohomologous to a constant and $m_{u_{0}}\left(\delta l_{0}\right)=0$, then $h^{\prime}\left(T, m_{u}\right)=0$ and

$$
\begin{gathered}
h^{\prime \prime}\left(T_{0}, m_{u_{0}}\right)=-\operatorname{Var}_{u_{0}}^{T_{0}}\left(\delta u_{0}\right)-2 h\left(T_{0}, m_{u_{0}}\right) \operatorname{Cov}_{u_{0}}^{T_{0}}\left(\delta l_{0}, \delta u_{0}\right) \\
-h\left(T_{0}, m_{u_{0}}\right) m_{u_{0}}\left(\delta^{2} l_{0}\right) .
\end{gathered}
$$

(C). Finally, if in addition to the hypothesis in (B), we have (a) $m_{u_{\varepsilon}}\left(\delta l_{\varepsilon}\right)=0$ for all $\varepsilon$ and (b) $m_{0}=m_{u_{0}}$, then

$$
\begin{aligned}
h_{\text {top }}^{\prime \prime}\left(T_{0}\right) & =\operatorname{Cov}_{m_{0}}^{T_{0}}\left(h_{\text {top }}\left(T_{0}\right) \delta l_{0}, \delta u_{0}+h_{\text {top }}\left(T_{0}\right) \delta l_{0}\right) . \\
h^{\prime \prime}\left(T_{0}, m_{u_{0}}\right) & =-\operatorname{Cov}_{m_{0}}^{T_{0}}\left(\delta u_{0}, \delta u_{0}+h_{\text {top }}\left(T_{0}\right) \delta l_{0}\right) \\
h_{\text {top }}^{\prime \prime}\left(T_{0}\right)-h^{\prime \prime}\left(T_{0}, m_{u_{0}}\right) & =\operatorname{Var}_{m_{0}}^{T_{0}}\left(\delta u_{0}+h_{\text {top }}\left(T_{0}\right) \delta l_{0}\right) .
\end{aligned}
$$

The formula (A1) was essentially a step of [KKW91] and (A2) has also been proved independently by Pollicott [Pol94].

The above proposition yields formulas for the second derivative of Ent $t_{\text {top }}$ and $\mathrm{Ent}_{\text {Liouv }}$ along paths $g_{\varepsilon}$ of metrics in $\mathcal{M}(M)$ once one knows how to determine the variations of the cocycles generating the Margulis and the Liouville measures-i.e. the length cocycle and the Liapunov cocycle-in terms of the variation $S=\frac{d}{d \varepsilon} g_{\varepsilon}$ of the Riemannian metric. In fact, since Gibbs states only depend on the cohomology class of the generating cocycles it is sufficient to determine the variation of the cohomology class of these cocycles. This is the second step of the proof.

1.3.2. Notation. For a symmetric covariant tensor field $S$ of rank 2 on a Riemannian manifold $\left(M, g_{0}\right)$, denote by $S^{\vee}$ the quadratic form field $S^{\vee}$ : $v \mapsto S(v, v)$ defined on the sphere bundle $S_{g_{0}} M$.

Then for the length cocycle we have $\delta l_{0}=\left.\frac{1}{2} S^{\vee}\right|_{\varepsilon=0}$. For the Liapunov cocycle we have the following Proposition:

1.3.3. Proposition. Let $g_{\varepsilon}$ be a $C^{1}$ curve of $C^{4}$ metrics on a manifold $M$, and assume that $g_{0}$ has constant negative curvature. Let $S=\frac{d}{d \varepsilon} g_{\varepsilon}$ at $\varepsilon=0$. 
Then the generator $\delta u$ of the first variation of the Liapunov cocycle at $\varepsilon=0$ is cohomologous to

$$
\delta u \approx T^{\vee}+\frac{1-n}{2} S^{\vee}
$$

where $T$ is the symmetric tensor field defined by $T=-\frac{1}{2} S+\frac{1}{4} \nabla^{*} \nabla S+$ $\frac{1}{2}\left(\operatorname{Tr}_{g} S\right) g-\frac{1}{2} \delta^{*} \delta S$.

In the Proposition above, $\nabla^{*} \nabla$ is the rough Laplacian for the metric $g_{0}, \delta^{*}$ denotes the symmetrization of the covariant derivative for $g_{0}$ and $\delta$ denotes its formal adjoint, the divergence.

The final step consists in estimating the covariance $\operatorname{Cov}_{m_{0}}\left(\delta l_{0}, \delta u_{0}\right)=$ $\operatorname{Cov}_{m_{0}}\left(\frac{1}{2} S^{\vee}, T^{\vee}+\frac{1-n}{2} S^{\vee}\right)$ which appears in the formulas (C1-3). We prove:

1.3.4. Proposition. Let $g_{0}$ be a metric on $M$ of constant negative curvature -1 . Let $S$ be a $C^{3}$ a symmetric covariant tensor field of rank 2 on $M$ and let $T=-\frac{1}{2} S+\frac{1}{4} \nabla^{*} \nabla S+\frac{1}{2}\left(\operatorname{Tr}_{g} S\right) g-\frac{1}{2} \delta^{*} \delta S$. Then, we have:

$$
\begin{gathered}
\operatorname{Cov}_{\operatorname{Liouv}\left(g_{0}\right)}^{g_{0}^{t}}\left(S^{\vee}, T^{\vee}\right) \geq \frac{n-2}{4} \operatorname{Cov}_{\operatorname{Liouv}\left(g_{0}\right)}^{g_{0}^{t}}\left(S^{\vee}, S^{\vee}\right) \\
\operatorname{Cov}_{\operatorname{Liouv}\left(g_{0}\right)}^{g_{t}^{t}}\left(T^{\vee}, T^{\vee}\right) \geq\left(\frac{n-2}{4}\right)^{2} \operatorname{Cov}_{\operatorname{Liouv}\left(g_{0}\right)}^{g_{0}^{t}}\left(S^{\vee}, S^{\vee}\right)
\end{gathered}
$$

The above estimate is the heart of the proof. It follows from observing that since $\left(M, g_{0}\right)$ is locally symmetric of constant negative curvature, the functions $S^{\vee}, T^{\vee}$ on the unit tangent bundle $S_{g_{0}} M$ lift to the orthonormal frame bundle $F M$ of $M$. The group $G \approx S O_{0}(1, n)$ acts transitively on $F M$, and the bilinear form $\operatorname{Cov}_{\operatorname{Liouv}\left(g_{0}\right)}^{g_{0}^{t}}$ is diagonal with respect to this action of $G$, i.e. it respects the decomposition of $L^{2}(F M)$ into irreducible subspaces. But the linear map $S \mapsto T$ also respects this decomposition and, on each irreducible subspace, $T$ is a multiple of $S$. In the end, the estimate reduces to an estimate of the smallest eigenvalue of the operator $S \mapsto T$ on the space orthogonal to the orbit of $g_{0}$ under $\operatorname{Diff}(M)$. This is achieved via a suitable Weitzenböck formula.

1.3.5. Corollary. Under the hypothesis of Theorem A and setting $S=\frac{d}{d \varepsilon} g_{\varepsilon}$ at $\varepsilon=0$, the second derivatives of the topological entropy and of the Liouville entropy satisfy:

$$
\begin{gathered}
\operatorname{Ent}_{\text {top }}^{\prime \prime}\left(g_{0}\right) \geq \frac{1}{4}\left(\begin{array}{c}
n-1 \\
2
\end{array}\right) \operatorname{Cov}_{\operatorname{Liouv}\left(g_{0}\right)}^{g_{0}^{t}}\left(S^{\vee}, S^{\vee}\right), \\
\operatorname{Ent}_{\text {top }}^{\prime \prime}\left(g_{0}\right)-\operatorname{Ent}_{\text {Liouv }}^{\prime \prime}\left(g_{0}\right) \geq \frac{(n-2)^{2}}{4} \operatorname{Cov}_{\operatorname{Liouv}\left(g_{0}\right)}^{g_{0}^{t}}\left(S^{\vee}, S^{\vee}\right),
\end{gathered}
$$


Proof of Theorems $A$ and $B$. The equality $\operatorname{Cov}_{\text {Liouv }\left(g_{0}\right)}^{g_{0}^{t}}\left(S^{\vee}, S^{\vee}\right)=0$, is equivalent to saying that $S^{\vee}$ is cohomologous to a constant, in our case to zero. Applying a theorem of Guillemin and Kazhdan [GK79], we obtain that this implies that the curve $g_{\varepsilon}$ is tangent to the orbit of the diffeomorphism group at $g_{0}$, in contradiction to the hypothesis. Thus $\operatorname{Cov}_{\text {Liouv }\left(g_{0}\right)}^{g_{0}^{t}}\left(S^{\vee}, S^{\vee}\right)>0$ and by Corollary 1.3.5 we obtain $\operatorname{Ent}_{\text {top }}^{\prime \prime}\left(g_{0}\right)>0$ and $\operatorname{Ent}_{\text {top }}^{\prime \prime}\left(g_{0}\right)-\operatorname{Ent}_{\text {Liouv }}^{\prime \prime}\left(g_{0}\right)>0$.

Acknowledgments. The largest part of this work was done at MSRI during the special year on "Lie Groups and Ergodic Theory". It is for me a pleasure to thank MSRI and the organizers of the program.

\section{$\S 2$. Derivatives of entropy for symbolic flows.}

\section{1.}

For generalities on topologically mixing subshifts of finite type, pressure and Gibbs states we refer to [Bow75] and [Rue78].

Let $\sigma: \Sigma \rightarrow \Sigma$ be a topologically mixing subshift of finite type. For any Hölder continuous potential $\phi$ denote by $\mu_{\phi}$ the Gibbs state for $\phi$, and by $P^{\sigma}(\phi)$ or $P(\sigma, \phi)$ the pressure of the potential $\phi$. We recall that pressure $P^{\sigma}(\phi)$ and the entropy $h\left(\sigma, \mu_{\phi}\right)$ of $\sigma$ with respect to the Gibbs state $\mu_{\phi}$ are related by the variational principle:

$$
P^{\sigma}(\phi)=h\left(\sigma, \mu_{\phi}\right)+\mu_{\phi}(\phi)=\sup _{\mu}(h(\sigma, \mu)+\mu(\phi)),
$$

where the supremum is taken as $\mu$ ranges on the set of probability $\sigma$-invariant measures on $\Sigma$. It is also known that, for $\phi \in C^{\alpha}(\Sigma)$, the equality $P^{\sigma}(\phi)=$ $h\left(\sigma, \mu_{\phi}\right)+\mu_{\phi}(\phi)$ completely characterizes the Gibbs state $\mu_{\phi}$ among the $\sigma$-invariant measures $\mu$ on $\Sigma$ (see [Bow75]).

We define the covariance of Hölder continuous functions $\psi$ and $\zeta$ with respect to the Gibbs state $\mu_{\phi}$ by

$$
\operatorname{Cov}_{\phi}^{\sigma}(\psi, \zeta)=\sum_{i=-\infty}^{\infty} \mu_{\phi}\left(\psi \cdot \zeta \circ \sigma^{i}-\mu_{\phi}(\psi) \mu_{\phi}(\zeta)\right)
$$

We also set

$$
\operatorname{Var}_{\phi}^{\sigma}(\psi)=\operatorname{Cov}_{\phi}^{\sigma}(\psi, \psi)
$$


and we call the above limit is called the variance of $\psi$ w.r.t. the Gibbs state $\mu_{\phi}$. We have $\operatorname{Cov}_{\phi}^{\sigma}(\psi, \zeta)=0$ for all $\psi$ if and only if $\zeta$ is cohomologous to a constant, i.e. $\zeta=\chi \circ \sigma-\chi+$ Const for some Hölder continuous function $\chi: \Sigma \rightarrow \mathbb{R}$.

The convergence of the above series follows from the exponential rate of mixing of Hölder continuous functions.

\section{2 .}

From [Con92] the map

$$
P^{\sigma}: \phi \in C^{\alpha}(\Sigma) \mapsto P^{\sigma}(\phi) \in \mathbb{R}
$$

is real analytic. Setting

$$
D^{n} P_{\phi_{0}}^{\sigma}\left(\phi_{1}, \ldots, \phi_{n}\right)=\left.\frac{d}{d t_{n}} \cdots \frac{d}{d t_{1}} P^{\sigma}\left(\phi_{0}+t_{1} \phi_{1}+\cdots+t_{n} \phi_{n}\right)\right|_{t_{1}=t_{2}=\cdots=t_{n}=0} .
$$

we have, (cf. [Con92] and [Rue78, Ch.5, Exerc. 5])

$$
D^{1} P_{\phi_{0}}^{\sigma}\left(\phi_{1}\right)=\mu_{\phi_{0}}\left(\phi_{1}\right) \quad \text { and } \quad D^{2} P_{\phi_{0}}^{\sigma}\left(\phi_{1}, \phi_{2}\right)=\operatorname{Cov}_{\phi_{0}}^{\sigma}\left(\phi_{1}, \phi_{2}\right) .
$$

Thus the maps

$$
\phi \in C^{\alpha}(\Sigma) \mapsto \mu_{\phi} \in C^{\alpha}(\Sigma)^{*}
$$

and

$$
\operatorname{Cov}^{\sigma}: \phi \in C^{\alpha}(\Sigma) \mapsto \operatorname{Cov}_{\phi}^{\sigma} \in L\left(C^{\alpha}(\Sigma), C^{\alpha}(\Sigma) ; \mathbb{R}\right)
$$

are real analytic.

2.2.1. Observation. If $\phi_{\varepsilon}$ is a $C^{2}$ curve of potentials in $C^{\alpha}(\Sigma)$, writing $\phi_{\varepsilon}=\phi_{0}+\varepsilon \phi_{1}+\frac{\varepsilon^{2}}{2} \phi_{2}+o\left(\varepsilon^{2}\right)$, we obtain

$$
P^{\sigma}\left(\phi_{\varepsilon}\right)=P^{\sigma}\left(\phi_{0}\right)+\varepsilon \mu_{\phi_{0}}\left(\phi_{1}\right)+\frac{\varepsilon^{2}}{2}\left(\operatorname{Cov}_{\phi_{0}}^{\sigma}\left(\phi_{1}, \phi_{1}\right)+\mu_{\phi_{0}}\left(\phi_{2}\right)\right)+o\left(\varepsilon^{2}\right) .
$$

\section{3.}

Let $\sigma: \Sigma \rightarrow \Sigma$ be a topologically mixing subshift of finite type and $\lambda$ be a positive $C^{\alpha}$ function on $\Sigma$. 
We recall the definition of special flow $T$ built under $\lambda$. Let $\tilde{T}$ be the flow on $\Sigma \times \mathbb{R}$ defined by $\tilde{T}^{t}(p, s)=(p, s+t)$ and consider on $\Sigma \times \mathbb{R}$ the equivalence relation $\sim$ generated by $(p, t) \sim(\sigma p, t-\lambda(p))$. Then, setting $X=\Sigma \times \mathbb{R} / \sim$, the flow $\tilde{T}$ descends to a flow $T$ on $X$ : the flow $(X, T)$ is called the special flow built under $\lambda$. Sometimes we shall simply write $(\Sigma, \sigma, \lambda, T)$ or $(\Sigma, \sigma, \lambda)$ to refer to $(X, T)$. Clearly a fundamental domain for $X$ is the set $\{(p, t) \in \Sigma \times \mathbb{R}: 0 \leq t<\lambda(p)\}$ and $X$ can be easily turned in a metric space (see $[\mathrm{BW} 72]$ ).

2.3.1. Notation. Let $A: X \rightarrow \mathbb{R}$ be a $C^{\alpha}$ continuous potential on $X$ and let $P^{T}(A)=P(T, A), m_{A}$ and $h\left(T, m_{A}\right)$ denote respectively the pressure of $A$ for the flow $T$, the Gibbs state for the potential $A$ and the entropy of the flow $T$ with respect to the measure $m_{A}$.

In analogy to the case of shifts, the pressure $P^{T}(A)$ and the entropy $h\left(T, m_{A}\right)$ of $T$ with respect to the Gibbs state $m_{A}$ are related by the variational principle:

$$
P^{T}(A)=h\left(T, m_{A}\right)+m_{A}(A)=\sup _{m}(h(T, m)+m(A)),
$$

where the supremum is taken as $m$ ranges on the set of probability $T$ invariant measures on $X$. Also, $m_{A}$ is the unique $T$-invariant measure on $X$ for which the equality $P^{T}(A)=h\left(T, m_{A}\right)+m_{A}(A)$ is achieved.

2.3.2. Definition. Let $c$ be the cocycle for the special flow $(X, T)=$ $(\Sigma, \sigma, \lambda)$ generated by $A: X \rightarrow \mathbb{R}$. Then the induced cocycle on $\Sigma$ is the cocycle for $\sigma$ generated by $\mathrm{I}[A]: \Sigma \rightarrow \mathbb{R}$ where $\mathrm{I}[A]$ is defined by

$$
\mathrm{I}[A](p)=\int_{0}^{\lambda(p)} A\left(T^{t} p\right) d t .
$$

2.3.3. Notation. Given a function $A: X \rightarrow \mathbb{R}$ let $\Phi[A]: \Sigma \rightarrow \mathbb{R}$ be the function defined by

$$
\Phi[A]=\mathrm{I}[A]-P^{T}(A) \lambda=\mathrm{I}\left[A-P^{T}(A)\right]
$$

If $A: X \rightarrow \mathbb{R}$ is a Hölder continuous on $X$, then $\mathrm{I}[A]$ and $\Phi[A]$ are also Hölder continuous on $\Sigma$.

We can reduce the study of flows to the case of shifts by the following theorem that clarifies the relation between Gibbs states for $(\Sigma, \sigma)$ and $(X, T)$. 
2.3.4. Theorem (Bowen, Franco-Sanchez). The Gibbs measure $m_{A}$ for the flow $T$ and the potential $A$ is given by

$$
m_{A}=\frac{\mu_{\Phi[A]} \otimes d t}{\left(\mu_{\Phi[A]} \otimes d t\right)(X)}=\frac{\mu_{\Phi[A]} \otimes d t}{\mu_{\Phi[A]}(\lambda)}
$$

where $\mu_{\Phi[A]}$ denotes the Gibbs state of the potential $\Phi[A]$ on $\Sigma$. Furthermore the pressure $P^{\sigma}(\Phi[A])$ of $\Phi[A]$ is zero.

2.3.5. Remark. From the above Theorem, Contreras' result mentioned in 2.2 , the formula 2.1 for the first derivative for $P^{T}(A)$ and the implicit function theorem, it follows that for $\lambda \in C^{\beta}(\Sigma)$ and $\alpha \leq \beta$, the map

$$
P^{T}: A \in C^{\alpha}(X) \mapsto P^{T}(A) \in \mathbb{R}
$$

is real analytic. The maps $m: A \in C^{\alpha}(X) \mapsto m_{A} \in C^{\alpha}(X)^{*}$ and $A \in$ $C^{\alpha}(X) \mapsto h\left(T, m_{A}\right) \in \mathbb{R}$ are real analytic as well.

2.3.6. Remark. The variational characterization of Gibbs states implies that the measure of maximal entropy for the flow $T$ is the Gibbs state for the potential zero (or, more generally, for a potential cohomologous to a constant).

2.3.7. Remark. Again by the variational principle, the potential $A$ has pressure zero if and only if

$$
h\left(T, m_{A}\right)=-m_{A}(A)
$$

\section{4 .}

We recall now the definition of covariance for special flows. Since an exponential mixing rate for Hölder functions is not guaranteed, the definition is not as immediate as in the case of shifts.

2.4.1. Notation. Retaining the previous notations let $B: X \rightarrow \mathbb{R}$ be another Hölder continuous function on $X$. Define $\Psi_{A}[B]: \Sigma \rightarrow \mathbb{R}$ by setting

$$
\Psi_{A}[B]=\mathrm{I}[B]-m_{A}(B) \lambda=\mathrm{I}\left[B-m_{A}(B)\right] .
$$

The definition of covariance was given by Marina Ratner who proved in [Rat73a] the following theorem: 
2.4.2. Theorem (Ratner). For a Hölder continuous function $B: X \rightarrow \mathbb{R}$ and any Gibbs measure $m_{A}$ on $X$ the limit

$$
\operatorname{Var}_{A}^{T}(B)=\lim _{T \rightarrow \infty} \frac{1}{T} \int_{X}\left(\int_{0}^{T}\left(B \circ T^{t}-m_{A}(B)\right) d t\right)^{2} d m_{A}
$$

exists, equals $\frac{1}{\mu_{\Phi[A]}(\lambda)} \operatorname{Var}_{\Phi[A]}^{\sigma}\left(\Psi_{A}[B]\right)$ and it is called the variance of $B$ with respect to the Gibbs state $m_{A}$.

By polarization, given any three Hölder continuous functions $A, B, C$ : $X \rightarrow \mathbb{R}$, the limit

$$
\begin{aligned}
& \operatorname{Cov}_{A}^{T}(B, C)=\lim _{T \rightarrow \infty} \frac{1}{T} \int_{X}\left(\int_{0}^{T}\left(B \circ T^{t}-m_{A}(B)\right) d t\right) \\
& \cdot\left(\int_{0}^{T}\left(C \circ T^{t^{\prime}}-m_{A}(C)\right) d t^{\prime}\right) d m_{A}
\end{aligned}
$$

exists and we have

$$
\operatorname{Cov}_{A}^{T}(B, C)=\frac{1}{\mu_{\Phi[A]}(\lambda)} \operatorname{Cov}_{\Phi[A]}^{\sigma}\left(\Psi_{A}[B], \Psi_{A}[C]\right) .
$$

We call $\operatorname{Cov}_{A}^{T}(B, C)$ the covariance of $B$ and $C$ with respect to the Gibbs state $m_{A}$. We have $\operatorname{Cov}_{A}^{T}(B, C)=0$ for all $B$ if and only if $C$ is cohomologous to a constant, i.e. if there exists a Hölder function $D$ differentiable along the flow $T^{t}$ such that $C=\frac{d D \circ T^{t}}{d t}{ }_{t=0}+$ Const (see [Rat73a]).

2.4.3. Remark. Notice that if the integral $\int_{-\infty}^{\infty} \mid m_{A}\left(B \cdot C \circ T^{t}\right)-$ $m_{A}(B) m_{A}(C) \mid d t$ exists, then

$$
\operatorname{Cov}_{A}^{T}(B, C)=\int_{-\infty}^{\infty}\left(m_{A}\left(B \cdot C \circ T^{t}\right)-m_{A}(B) m_{A}(C)\right) d t .
$$

\section{5.}

Let $\lambda_{\varepsilon}$ be a $C^{r}$ curve of positive $C^{\alpha}$ functions on $\Sigma$ and let $\left(X_{\varepsilon}, T_{\varepsilon}\right)$ be the family of special flows built under $\lambda_{\varepsilon}$.

Let $u_{\varepsilon}$ be the a $C^{r}$ family of $C^{\alpha}$ potentials on $X_{\varepsilon}$, i.e. a curve admitting a $C^{r}$ lift to $C^{\alpha}(\Sigma \times \mathbb{R})$. 
2.5.1. Definition. For $\lambda_{\varepsilon}$ and $u_{\varepsilon}$ as above we define the $i$-th variation of the cocycle (generated by) $u_{\varepsilon}$ as (the cocycle generated by) any $C^{\alpha}$ function $\delta^{i} u_{\varepsilon}$ be a on $X_{\varepsilon}$ with the property that

$$
\mathrm{I}_{\varepsilon}\left[\delta^{i} u_{\varepsilon}\right](p)=\int_{0}^{\lambda_{\varepsilon}(p)} \delta^{i} u_{\varepsilon}(p, t) d t=\frac{d^{i}}{d \varepsilon^{i}} \int_{0}^{\lambda_{\varepsilon}(p)} u_{\varepsilon}(p, t) d t .
$$

(for simplicity, $\delta^{1}=\delta$ and $\delta^{0} u_{\varepsilon}=u_{\varepsilon}$ ). In other words the $i$-th variation $\delta^{i} u_{\varepsilon}$ induces on $\Sigma$ the $i$-th derivative of the induced cocycle $\mathrm{I}_{\varepsilon}\left[u_{\varepsilon}\right]$. Notice that the $i$-th variation $\delta^{i} u_{\varepsilon}$ of the cocycle $u_{\varepsilon}$ is only defined up to a coboundary for the flow $T_{\varepsilon}$.

We denote by $\delta^{i} l_{\varepsilon}$, the $i$-th variation of the length cocycle:

$$
\mathrm{I}_{\varepsilon}\left[\delta^{i} l_{\varepsilon}\right](p)=\int_{0}^{\lambda_{\varepsilon}(p)} \delta^{i} l_{\varepsilon}(p, t) d t=\frac{d^{i}}{d \varepsilon^{i}} \lambda_{\varepsilon}(p) .
$$

Having stated the set up the proof of Proposition 1.3.1 is rather elementary. To simplify notation, we denote with primes differentiation with respect to $\varepsilon$ and suppress the dependence on $\varepsilon$, e.g. $P^{\prime}(T, u)=d / d \varepsilon P\left(T_{\varepsilon}, u_{\varepsilon}\right)$ and $P^{\prime}\left(T_{0}, u_{0}\right)=d /\left.d \varepsilon P\left(T_{\varepsilon}, u_{\varepsilon}\right)\right|_{\varepsilon}=0$.

\subsubsection{Proposition (Derivatives of the Pressure and Entropy). Let} $\lambda_{\varepsilon}$ be a $C^{2}$ curve of positive $C^{\alpha}$ functions on $\Sigma$ and let $\left(X_{\varepsilon}, T_{\varepsilon}\right)$ be the family of special flows built under $\lambda_{\varepsilon}$. Let $u_{\varepsilon}$ be the a $C^{2}$ family of $C^{\alpha}$ potentials on $X_{\varepsilon}$. Then, retaining the previous notation for the first and second variation for length cocycle and the cocycle generated by $u_{\varepsilon}$ and setting

$$
v_{\varepsilon}=\delta u_{\varepsilon}-P\left(T_{\varepsilon}, u_{\varepsilon}\right) \delta l_{\varepsilon} \quad \text { and } \quad w_{\varepsilon}=\delta^{2} u_{\varepsilon}-P\left(T_{\varepsilon}, u_{\varepsilon}\right) \delta^{2} l_{\varepsilon}
$$

we have:

$$
\begin{aligned}
P^{\prime}(T, u) & =m_{u}(v) \\
P^{\prime \prime}(T, u) & =\operatorname{Var}_{u}^{T}(v)+m_{u}(w)-2 m_{u}(v) m_{u}(\delta l)
\end{aligned}
$$

and

$$
\begin{array}{r}
h^{\prime}\left(T, m_{u}\right)=-\operatorname{Cov}_{u}(v, u)+h\left(T, m_{u}\right) m_{u}(\delta l) \\
h^{\prime \prime}\left(T, m_{u}\right)=-D^{3} P_{u}^{T}(u, v, v)-2 h\left(T, m_{u}\right) \operatorname{Cov}_{u}^{T}(\delta l, v) \\
+2\left(\operatorname{Cov}_{u}^{T}(v, u)+h\left(T, m_{u}\right) m_{u}(\delta l)\right) m_{u}(\delta l) \\
-\operatorname{Cov}_{u}^{T}(u, w)+2 m_{u}(v) \operatorname{Cov}_{u}^{T}(u, \delta l) \\
-h\left(T, m_{u}\right) m_{u}\left(\delta^{2} l\right)-\operatorname{Cov}_{u}^{T}(v, v) .
\end{array}
$$


We shall not give the proof of the proposition above. However, for completeness, we shall give independent proofs of the following corollaries which we use in this paper.

2.6.1. Corollary. Let $\lambda_{\varepsilon}$ be a $C^{2}$ curve of positive $C^{\alpha}$ functions on $\Sigma$ and let $\left(X_{\varepsilon}, T_{\varepsilon}\right)$ be the family of special flows built under $\lambda_{\varepsilon}$. We retain here the previous notation and denote by $h_{\mathrm{top}}\left(T_{\varepsilon}\right)$ the topological entropy of the flow $T_{\varepsilon}$ and by $M_{\varepsilon}$ the measure of maximal entropy for the flow $T_{\varepsilon}$. Also denote by $\operatorname{Var}_{0}^{T_{\varepsilon}}$ the variance of the flow $T_{\varepsilon}$ with respect to the measure of maximal entropy $m_{\varepsilon}$. Then, suppressing the dependence on $\varepsilon$, we have

$$
h_{\mathrm{top}}^{\prime}(T)=-h_{\mathrm{top}}(T) M(\delta l) .
$$

If $h_{\mathrm{top}}^{\prime}\left(T_{0}\right)=0$ we have

$$
h_{\text {top }}^{\prime \prime}\left(T_{0}\right)=-h_{\text {top }}\left(T_{0}\right) M_{0}\left(\delta^{2} l_{0}\right)+h_{\text {top }}\left(T_{0}\right)^{2} \operatorname{Var}_{0}^{T_{0}}\left(\delta l_{0}\right) .
$$

Proof. Observe that since $\varepsilon \mapsto \lambda_{\varepsilon} \in C^{\alpha}(\Sigma)$ is $C^{2}$, the first and second variation $\delta l_{\varepsilon}$ and $\delta^{2} l_{\varepsilon}$ of the length cocycle exist (as Hölder functions). By the Remark 2.3.6 the topological entropy equals the pressure for the potential 0 and from Theorem 2.3.4 and 2.3 we obtain that

$$
m_{\varepsilon}=\frac{\mu_{\phi(\varepsilon)} \otimes d t}{\mu_{\phi(\varepsilon)}\left(\lambda_{\varepsilon}\right)}
$$

where $\phi(\varepsilon)=-h_{\text {top }}\left(T_{\varepsilon}\right) \lambda_{\varepsilon}$. Again by Theorem 2.3.4, we have that $P^{\sigma}(\phi(\varepsilon))=0$ for all $\varepsilon$. By Remark 2.3.5, the curve $\phi(\varepsilon)$ is a $C^{2}$ curve of $C^{\alpha}$ potentials on $\Sigma$. Thus from (2.2) we obtain that

$$
\mu_{\phi}\left(\phi^{\prime}\right)=0 \quad \text { and } \quad \operatorname{Var}_{\phi}^{\sigma}\left(\phi^{\prime}\right)+\mu_{\phi}\left(\phi^{\prime \prime}\right)=0 .
$$

Since $\phi^{\prime}=-h_{\mathrm{top}}^{\prime}(T) \lambda-h_{\mathrm{top}}(T) \lambda^{\prime}$ from the first of (2.9) we conclude that

$$
h_{\mathrm{top}}^{\prime}(T)=-\frac{h_{\mathrm{top}}(T) \mu_{\phi}\left(\lambda^{\prime}\right)}{\mu_{\phi}(\lambda)}=-\frac{h_{\mathrm{top}}(T) \mu_{\phi} \otimes d t(\delta l)}{\mu_{\phi}(\lambda)}=-h_{\mathrm{top}}(T) m_{0}(\delta l)
$$

proving (2.7).

The hypothesis $h_{\text {top }}^{\prime}\left(T_{0}\right)=0$ implies that $\phi^{\prime}(0)=-h_{\text {top }}^{\prime}\left(T_{0}\right) \lambda_{0}$ and $\phi^{\prime \prime}(0)=-h_{\text {top }}^{\prime \prime}\left(T_{0}\right) \lambda_{0}-h_{\text {top }}\left(T_{0}\right) \lambda_{0}^{\prime \prime}$. Thus, using the second of the formulas $(2.9)$, we obtain

$$
\operatorname{Var}_{\phi(0)}^{\sigma}\left(-h_{\text {top }}\left(T_{0}\right) \lambda_{0}^{\prime}\right)+\mu_{\phi(0)}\left(-h_{\text {top }}^{\prime \prime}\left(T_{0}\right) \lambda_{0}-h_{\text {top }}\left(T_{0}\right) \lambda_{0}^{\prime \prime}\right)=0 .
$$


We conclude that

$$
\begin{gathered}
h_{\text {top }}^{\prime \prime}\left(T_{0}\right)=-h_{\text {top }}\left(T_{0}\right) \frac{\mu_{\phi(0)}\left(\lambda_{0}^{\prime \prime}\right)}{\mu_{\phi(0)}\left(\lambda_{0}\right)}+h_{\text {top }}\left(T_{0}\right)^{2} \frac{\operatorname{Var}_{\phi_{0}}^{\sigma}\left(\lambda_{0}^{\prime}\right)}{\mu_{\phi(0)}\left(\lambda_{0}\right)} \\
=-h_{\text {top }}\left(T_{0}\right) M_{0}\left(\delta l_{0}\right)+h_{\text {top }}\left(T_{0}\right)^{2} \frac{\operatorname{Var}_{\phi_{0}}^{\sigma}\left(\mathrm{I}\left[\delta \lambda_{0}\right]\right)}{\mu_{\phi(0)}\left(\lambda_{0}\right)}
\end{gathered}
$$

From $h_{\text {top }}^{\prime}\left(T_{0}\right)=0$ we have $M_{0}\left(\delta l_{0}\right)=0$ and therefore $\mathrm{I}\left[\delta \lambda_{0}\right]=\Psi_{0}\left[l_{0}^{\prime}\right]$. Using (2.4) we conclude

$$
\frac{\operatorname{Var}_{\phi_{0}}^{\sigma}\left(\mathrm{I}\left[\delta \lambda_{0}\right]\right)}{\mu_{\phi(0)}\left(\lambda_{0}\right)}=\operatorname{Var}_{m_{0}}^{T^{t}}\left(\delta l_{0}\right)
$$

proving (2.8) and Part A of Proposition 1.3.1.

2.6.2. Corollary. Let $\lambda_{\varepsilon}$ be a $C^{2}$ curve of positive $C^{\alpha}$ functions on $\Sigma$ and let $\left(X_{\varepsilon}, T_{\varepsilon}\right)$ be the family of special flows built under $\lambda_{\varepsilon}$. Let $u_{\varepsilon}$ be the a $C^{2}$ family of $C^{\alpha}$ potentials on $X_{\varepsilon}$. In the hypothesis that $P\left(T_{\varepsilon}^{t}, u_{\varepsilon}\right)=0$ for all $\varepsilon$ we have:

$$
h^{\prime}\left(T, m_{u}\right)=-\operatorname{Cov}_{u}^{T}(u, \delta u)-h\left(T, m_{u}\right) m_{u}(\delta l) .
$$

If $u_{0}$ is cohomologous to a constant and $m_{u_{0}}\left(\delta l_{0}\right)=0$, then $h^{\prime}\left(T, m_{u}\right)=0$ and

$$
\begin{aligned}
h^{\prime \prime}\left(T_{0}, m_{u_{0}}\right)=-\operatorname{Var}_{u_{0}}^{T_{0}}\left(\delta u_{0}\right)-2 h\left(T_{0}, m_{u_{0}}\right) & \operatorname{Cov}_{u_{0}}^{T_{0}}\left(\delta l_{0}, \delta u_{0}\right) \\
& -h\left(T_{0}, m_{u_{0}}\right) m_{u_{0}}\left(\delta^{2} l_{0}\right)
\end{aligned}
$$

Proof. Since by hypothesis $P\left(T_{\varepsilon}^{t}, u_{\varepsilon}\right)=0$ for all $\varepsilon$, we have that $\Phi\left[u_{\varepsilon}\right](p)=$ $\int_{0}^{\lambda_{\varepsilon}(p)} u_{\varepsilon}\left(T_{\varepsilon}^{t} p\right) d t$. For simplicity set $\psi_{\varepsilon}=\Phi\left[u_{\varepsilon}\right]$. The Gibbs state $m_{u_{\varepsilon}}$ is thus given by

$$
m_{u_{\varepsilon}}=\frac{\mu_{\psi_{\varepsilon}} \otimes d t}{\mu_{\psi_{\varepsilon}}\left(\lambda_{\varepsilon}\right)}
$$

where $\mu_{\psi_{\varepsilon}}$ denotes as before the Gibbs measure for the shift $\sigma$ and the potential $\psi_{\varepsilon}$. From equation 2.2 and the fact that $P\left(\sigma, \psi_{\varepsilon}\right)=0$ for all $\varepsilon$ (see 2.2 ), we obtain

$$
\mu_{\psi}\left(\psi^{\prime}\right)=0
$$

By the variational characterization of Gibbs states and $P\left(\sigma, \psi_{\varepsilon}\right)=0$ we have $h\left(\sigma, \mu_{\psi}\right)=-\mu_{\psi}(\psi)$ and, using (2.12), we obtain

$$
h^{\prime}\left(\sigma, \mu_{\psi}\right)=-\operatorname{Cov}_{\psi}^{\sigma}\left(\psi, \psi^{\prime}\right)-\mu_{\psi}\left(\psi^{\prime}\right)=-\operatorname{Cov}_{\psi}^{\sigma}\left(\psi, \psi^{\prime}\right)
$$


Differentiating w.r.t. $\varepsilon$ Abramov's formula

$$
h\left(\sigma, \mu_{\psi_{\varepsilon}}\right)=\mu_{\psi_{\varepsilon}}\left(\lambda_{\varepsilon}\right) h\left(T_{\varepsilon}, m_{u_{\varepsilon}}\right)
$$

and using (2.13), we have

$$
-\operatorname{Cov}_{\psi}^{\sigma}\left(\psi, \psi^{\prime}\right)=h\left(T, m_{u}\right)\left(\operatorname{Cov}_{\psi}^{\sigma}\left(\lambda, \psi^{\prime}\right)+\mu_{\psi_{\varepsilon}}\left(\lambda^{\prime}\right)\right)+\mu_{\psi_{\varepsilon}}(\lambda) h^{\prime}\left(T, m_{u}\right)
$$

or

$$
h^{\prime}\left(\sigma, m_{u}\right)=-\frac{\operatorname{Cov}_{\psi}^{\sigma}\left(\psi+h\left(T, m_{u}\right) \lambda, \psi^{\prime}\right)}{\mu_{\psi_{\varepsilon}}(\lambda)}-h\left(T, m_{u}\right) \frac{\mu_{\psi_{\varepsilon}}\left(\lambda^{\prime}\right)}{\mu_{\psi_{\varepsilon}}(\lambda)} .
$$

Observe that we have $\Psi_{u}[u]=\psi-m_{u}(u) \lambda=\psi+h\left(T, m_{u}\right) \lambda$ and $\Psi_{u}[\delta u]=$ $\psi^{\prime}-m_{u}(\delta u) \lambda=\psi^{\prime}-\mu_{\psi}\left(\psi^{\prime}\right) \lambda=\psi^{\prime}$. Thus, using (2.4) we have $\operatorname{Cov}_{\psi}^{\sigma}(\psi+$ $\left.h\left(T, m_{u}\right) \lambda, \psi^{\prime}\right)=\operatorname{Cov}_{u}^{T}(u, \delta u) \mu_{\psi_{\varepsilon}}(\lambda)$, which proves (2.10).

Assume now that the $u_{0}$ is cohomologous to a constant. Then $P(T, u)=$ 0 implies that the function $u_{0}+h\left(T_{0}, m_{u_{0}}\right)$ is cohomologous to zero and therefore also $\psi_{0}+h\left(m_{u_{0}}\right) \lambda_{0}$ is cohomologous to a zero. The further assumption $m_{u_{0}}\left(\delta l_{0}\right)=0$ entails $\mu_{\psi_{0}}\left(\lambda_{0}^{\prime}\right)=0$ and therefore $h^{\prime}\left(T_{0}, m_{u_{0}}\right)=0$. In this case one further differentiation of (2.14) yields

$$
\begin{aligned}
h^{\prime \prime}\left(m_{u_{0}}\right)=- & \frac{\operatorname{Cov}_{\psi_{0}}^{\sigma}\left(\psi_{0}^{\prime}+h\left(m_{u_{0}}\right) \lambda_{0}^{\prime}, \psi_{0}^{\prime}\right)}{\mu_{\psi_{0}}\left(\lambda_{0}\right)}- \\
& -h\left(m_{u_{0}}\right)\left[\frac{\operatorname{Cov}_{\psi_{0}}^{\sigma}\left(\psi_{0}^{\prime}, \lambda_{0}^{\prime}\right)}{\mu_{\psi_{0}}\left(\lambda_{0}\right)}+\frac{\mu_{\psi_{0}}\left(\lambda_{0}^{\prime \prime}\right)}{\mu_{\psi_{0}}\left(\lambda_{0}\right)}\right] \\
=- & \frac{\operatorname{Cov}_{\psi_{0}}^{\sigma}\left(\psi_{0}^{\prime}, \psi_{0}^{\prime}\right)}{\mu_{\psi_{0}}\left(\lambda_{0}\right)}-h\left(m_{u_{0}}\right)\left[2 \frac{\operatorname{Cov}_{\psi_{0}}^{\sigma}\left(\psi_{0}^{\prime}, \lambda_{0}^{\prime}\right)}{\mu_{\psi_{0}}\left(\lambda_{0}\right)}+\frac{\mu_{\psi_{0}}\left(\lambda_{0}^{\prime \prime}\right)}{\mu_{\psi_{0}}\left(\lambda_{0}\right)}\right] .
\end{aligned}
$$

and (2.11) is proved. Part B of Proposition 1.3.1 is now proved.

2.6.3. Corollary. Let $\lambda_{\varepsilon}$ be a $C^{2}$ curve of positive $C^{\alpha}$ functions on $\Sigma$ and let $\left(X_{\varepsilon}, T_{\varepsilon}\right)$ be the family of special flows built under $\lambda_{\varepsilon}$. Let $u_{\varepsilon}$ be the a $C^{2}$ family of $C^{\alpha}$ potentials on $X_{\varepsilon}$. Assume that

1. $P\left(T_{\varepsilon}^{t}, u_{\varepsilon}\right)=0$ for all $\varepsilon$;

2. $m_{u_{\varepsilon}}\left(\delta l_{\varepsilon}\right)=0$ for all $\varepsilon$;

3. $u_{0}$ is cohomologous to a constant. 
Then $m_{0}=m_{u_{0}}$, then we obtain $m_{0}\left(\delta^{2} l_{0}\right)=-\operatorname{Cov}_{m_{0}}^{T_{0}}\left(\delta l_{0}, \delta u_{0}\right)$. Therefore we obtain $h_{\mathrm{top}}^{\prime}\left(T_{0}\right)=h^{\prime}\left(T_{0}, m_{u_{0}}\right)=0$ and

$$
\begin{aligned}
h_{\text {top }}^{\prime \prime}\left(T_{0}\right) & =\operatorname{Cov}_{m_{0}}\left(h_{\text {top }}\left(T_{0}\right) \delta l_{0}, u_{0}^{\prime}+h_{\text {top }}\left(T_{0}\right) \delta l_{0}\right) . \\
h^{\prime \prime}\left(T_{0}, m_{u_{0}}\right) & =-\operatorname{Cov}_{m_{0}}\left(u_{0}^{\prime}, u_{0}^{\prime}+h_{\text {top }}\left(T_{0}\right) \delta l_{0}\right) \\
h_{\text {top }}^{\prime \prime}\left(T_{0}\right)-h^{\prime \prime}\left(T_{0}, m_{u_{0}}\right) & =\operatorname{Cov}_{m_{0}}\left(u_{0}^{\prime}+h_{\text {top }}\left(T_{0}\right) \delta l_{0}, u_{0}^{\prime}+h_{\text {top }}\left(T_{0}\right) \delta l_{0}\right)
\end{aligned}
$$

Proof. The claim $m_{0}=m_{u_{0}}$ is equivalent to $u_{0}$ being cohomologous to a constant. Retain the notation of the proof of the previous Corollary. Since $m_{u_{\varepsilon}}\left(\delta l_{\varepsilon}\right)=0$ implies $\mu_{\psi_{\varepsilon}}\left(\lambda_{\varepsilon}^{\prime}\right)=0$ for all $\varepsilon$, differentiating we obtain

$$
\operatorname{Cov}_{\psi_{\varepsilon}}^{\sigma}\left(\lambda_{\varepsilon}^{\prime}, \psi_{\varepsilon}^{\prime}\right)+\mu_{\psi_{\varepsilon}}\left(\lambda_{\varepsilon}^{\prime \prime}\right)=0 .
$$

From $m_{u_{\varepsilon}}\left(\lambda^{\prime}\right)=0$ we obtain $\Psi_{u_{\varepsilon}}[\delta l]=\lambda^{\prime}$ and since $\Psi_{u_{\varepsilon}}[\delta u]=\psi^{\prime}$, using 2.4, we obtain that

$$
m_{u}\left(\delta^{2} \lambda\right)=-\operatorname{Cov}_{u}^{T}(\delta l, \delta u) .
$$

The rest is now mere rephrasing. This also concludes the proof of Proposition 1.3.1.

\section{$\S 3$. The first variation of the length and Liapunov cocycles.}

In this section we collect some well known facts that we allow us to connect the results of $\S 2$ to the study of our original problem. Some of the theorems stated are valid in a more general setting of Anosov or Axiom-A flows. We state them for the case of geodesic flows for metrics of negative curvature.

\subsection{Symbolic Coding.}

The geodesic flow $T_{g}$ of a metric of negative curvature $g$ on the unit tangent bundle $S_{g} M$ of a manifold $M$ is isomorphic to a symbolic flow [Rat69], [Rat73b], [Bow72b]. More exactly, there is symbolic flow $(X, T)$ built over a topologically mixing subshift of finite type $(\Sigma, \sigma)$ with a positive Hölder continuous ceiling function $\lambda$ and a finite-to-one Hölder continuous surjection $\pi: X \rightarrow S_{g} M$ such that $\pi$ intertwines the flow $T$ on $X$ with the flow $T_{g}$ on $S_{g} M$. Furthermore, denoting by $m_{u}$ and $n_{u \circ \pi}$ respectively the Gibbs state for $u \in C^{\alpha}\left(S_{g} M\right)$ with respect to the flow $T_{g}$ and Gibbs state for $u \circ \pi \in C^{\beta}(X)$ with respect to the flow $T$, we have that the surjection 
$\pi$ is a measure theoretical isomorphism of $\left(X, T, n_{u \circ \pi}\right)$ onto $\left(S_{g}, T_{g}, m_{u}\right)$. In particular $u \in C^{\alpha}\left(S_{g} M\right)$ is cohomologous to zero if and only if $u \circ \pi$ is cohomologous to zero.

The Margulis measure and the Liouville measure on $S_{g} M$ are the Gibbs states respectively for the length cocycle and for the Liapunov cocycle. We recall the definition of Liapunov cocycle.

3.1.1. The Liapunov cocycle. For $v \in S_{g} M$ let $W^{s s}(v)$ denote the strong stable manifold passing through $v$, i.e. the set of points $w \in S_{g} M$ with $d\left(\phi^{t} v, \phi^{t} w\right) \rightarrow 0$ as $t \rightarrow+\infty$. The Liapunov cocycle is given by the growth of the volume of strong stable manifold: more exactly, if $\mu^{s s}$ is the volume form induced on strong stable manifolds by some Riemannian metric on $S_{g} M$, the Liapunov cocycle is defined by $\mathcal{L}(v, t)=\frac{d\left(\phi^{t}\right)^{*} \mu^{s s}}{d \mu^{s s}}(v)$. Changing the Riemannian metric on $S_{g} M$ does not affect the cohomology class of the Liapunov cocycle.

The Liapunov cocycle has an interpretation in terms of Jacobi fields. The natural projection $p: S_{g} M \rightarrow M$ maps bijectively the strong stable manifold $W_{g}^{s s}(v)$ onto the stable horosphere $\mathcal{H}_{g}(v)$ of $v$. The second fundamental form $U_{g}(v)$ of the stable horosphere $\mathcal{H}(v)$ at $p(v)$ considered as a tensor of type $\left(\begin{array}{l}1 \\ 1\end{array}\right)$ satisfies ${ }^{2}$ along the orbit $T_{g}^{t} v$ the Riccati equation

$$
\frac{\nabla}{d t} U_{g}\left(T_{g}^{t} v\right)+U_{g}^{2}\left(T_{g}^{t} v\right)=R\left(p_{*} T_{g}^{t} v, \cdot\right) p_{*} T_{g}^{t} v .
$$

The Liapunov cocycle can be expressed as

$$
\mathcal{L}_{g}(v, t)=\int_{0}^{t} \operatorname{Tr} U_{g}\left(T_{g}^{\tau} v\right) d \tau
$$

It is not difficult to see that $\operatorname{Tr} U_{g}$ is a Hölder continuous function on $S_{g} M$.

3.1.2. The construction yielding $(\Sigma, \sigma, \lambda)$ and the semi-conjugacy $\pi$, proceeds by choosing in $S_{g} M$ finitely many disjoint smooth disks $D_{i}$ transversal to the flow $T_{g}$. Inside each $D_{i}$, we choose closed connected sets $R_{i}$ so that each $R_{i}$ equals the closure of its interior $R_{i}^{\circ}$ and the union $R$ of all $R_{i}$ 's forms a cross-section to the flow $T_{g}$. Let $P$ be the Poincaré map of $R$. Then $\Sigma$ is obtained as the collection of sequences $\omega=\left(\omega_{i}\right)_{i \in Z}$ such that

\footnotetext{
${ }^{2}$ Technically the map $v \mapsto U_{g}(v)$ is a section of the pull-back to $S_{g} M$ of the bundle $T^{(1,1)} M \rightarrow M$ via the natural projection $p: S M \rightarrow M$. The Riccati equation can then be interpreted in these terms, $\nabla$ being the pull-back to $S_{g} M$ of the Levi-Civita connection on $M$.
} 
$\cap_{i \in Z} P^{-i} R_{\omega_{i}} \neq \varnothing$. By the expansiveness of the flow $T_{g}$ if $\omega \in \Sigma$ then the set $\cap_{i \in Z} P^{-i} R_{\omega_{i}}$ contains exactly one point $x=x(\omega)$ of $R$, thus defining a surjection $\pi_{\Sigma}: \omega \mapsto x(\omega)$. For each $p \in R$ the first return time $\bar{\lambda}(p)$ to $P$ under the Poincare map of $P$ is well defined and positive. Let $\lambda=\bar{\lambda} \circ \pi_{\Sigma}$ and let $\sigma$ be the shift on $\Sigma$ and define $(X, T)$ as the symbolic flow $(X, T)$ built over $(\Sigma, \sigma)$ with ceiling function $\lambda$. It is plain that $p_{\Sigma}$ extents to a a surjection $\pi: X \rightarrow S_{g} M$ intertwining the flow $T$ on $X$ with the flow $T_{g}$ on $S_{g} M$.

\subsection{Structural Stability.}

With $M$ a compact manifold, denote by $S M$ the bundle of oriented directions on $M$, i.e. $S M=\{v \in T M \mid v \neq 0\} / \sim$, where $v \sim v^{\prime}$ and if and only if $\exists c>0, v=c v^{\prime}$. For each Riemannian metric $g$ there is a natural identification between the bundle $S M$ and the unit tangent bundle $S_{g} M$. By means of this identification, we regard the geodesic flow $T_{g}$ for the metric $g$ as a flow on $S M$, which we also denote by $T_{g}$ with abuse of notation.

Denote by $\mathcal{U}$ the space of $C^{2}$-metrics of negative curvature on $M$, and more generally by $\mathcal{U}^{s}$ the space of $C^{s}$-metrics of negative curvature on $M$.

It is well known, (cf. [Mor24], [Ano67] and [Gro]), that the various flows $T_{g}$ for $g \in \mathcal{U}$ are all orbit-equivalent. This means that if $g_{0}$ and $g \in \mathcal{U}$, there is a homeomorphism $H_{g}: S M \rightarrow S M$ sending the orbits of the flow $T_{g_{0}}$ to the orbits of the flow $T_{g}$. In fact the homeomorphism $H_{g}$ is homotopic to the identity and can always be chosen smooth along the orbits of $T_{g_{0}}$ and Hölder continuous on $S M$. We call such an homeomorphism a $\left(g_{0}, g\right)$-Morse correspondence. The Morse correspondence is not unique: if $H_{1}$ and $H_{2}$ are two $\left(g_{0}, g\right)$-Morse correspondences then there exists a real valued function $t(v)$ on $S M$ such that $\forall v \in S M, H_{1}^{-1} \circ H_{2}(v)=T_{g_{0}}^{t(v)}(v)$. Thus we have a sort of transversal uniqueness:

3.2.1. Definition. For $g$ in a neighbourhood $\mathcal{V} \subseteq \mathcal{U}$, we will call the family of Morse correspondences normal at $g_{0}$, the family $g \mapsto H_{g}$ of $\left(g_{0}, g\right)$-Morse correspondences $H_{g}$ which is uniquely defined by the property

"for each $v \in S M$ the footpoint of $H_{g}(v)$ belongs to the hypersurface obtained by exponentiating in the metric $g_{0}$ a small ball in the subspace $g_{0}$-perpendicular to $v "$

We have described some of the generalities of the construction of the symbolic flow in order to understand what happens if we perturb the flow 
$T_{g_{0}}$ by perturbing a metric $g_{0} \in \mathcal{U}$. Let $\pi_{g_{0}}$ be a semi-conjugacy between a symbolic flow $\left(\Sigma, \sigma, \lambda_{g_{0}}\right)$ and $\left(S M, T_{g_{0}}\right)$ mentioned in Section 3.1 .

For $g$ in some subset of $\mathcal{U}$, denoting by $H_{g}$ the family of Morse correspondences normal at $g_{0}$, from the fact that $H_{g}$ sends the orbits of the flow $T_{g_{0}}$ to the orbits of the flow $T_{g}$, we obtain that the set $H_{g} \circ \pi_{g_{0}}(\Sigma)$ provides a global cross-section for the flow $T_{g}$ with a Hölder continuous return function $\lambda_{g}: \Sigma \rightarrow \mathbb{R}$

We obtain a finite-to-one Hölder continuous surjection $\pi_{g}: X_{g} \rightarrow S M$ intertwining the symbolic flow on $X_{g}=\Sigma \times \mathbb{R} /\left[\left(p, \lambda_{g}(p)+t\right) \sim(\sigma p, t)\right]$ and the flows $T_{g}$ on $S M$. [Equivalently we can look at the composition $H_{g} \circ \pi$ as giving an orbit equivalence between $\left(\Sigma, \sigma, \lambda_{g_{0}}\right)$ and $\left(S M, T_{g}\right)$.] Thus we have the following diagram

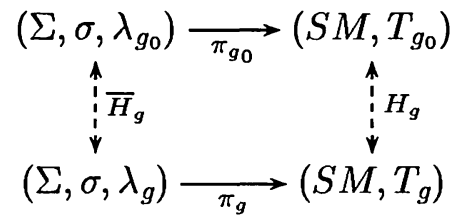

with dashed arrows representing orbit equivalences. Observe that, identifying the space $X_{g}$ supporting the flow $\left(\Sigma, \sigma, \lambda_{g}\right)$ with $X_{g}=\{(p, t) \in \Sigma \times \mathbb{R} \mid 0 \leq$ $\left.t<\lambda_{g}\right\}$ and $\Sigma$ with $\Sigma \times\{0\}$, the orbit equivalences $\bar{H}_{g}$ are the identity on $\Sigma$.

\subsection{Variation of cocycles.}

Now we are in the situation in which we can apply the results of $\S 2$. To this purpose we need to pull-back the length and the Liapunov cocycles to the flow $\left(\Sigma, \sigma, \lambda_{g}\right)$ or $\left(\Sigma, \sigma, \lambda_{g_{0}}\right)$, and determine in geometric terms their first variations $\delta l, \delta u$ which entered in Proposition 1.3.1. Finally we need to resolve the smoothness issues related to this construction.

Assume that for each $g \in \mathcal{U}$ we are given a cocycle $c_{g}$ for the flow $T_{g}$ and orbit equivalences $H_{g}: S M \rightarrow S M$ sending the orbits of the flow $T_{g_{0}}$ to the orbits of the flow $T_{g}$.

3.3.1. Definition. The pull-back of the family of cocycles $c_{g}$ along the orbit equivalences $H_{g}$ is the family of cocycles $H_{g}^{*} c_{g}$ for the flow $T_{g_{0}}$ given by

$$
H_{g}^{*} c_{g}(v, t)=c_{g}\left(H_{g} v, t^{\prime}\right)
$$

where $t^{\prime}$ is defined by $T_{g}^{t^{\prime}} H_{g} v=H_{g} T_{g_{0}}^{t} v$. 
3.3.2. Example. The length cocycles $\ell_{g}(v, t)=t$ for the flow $T_{g}$ is generated by $A_{g}=1$. If the orbit equivalences are differentiable along the flow $T_{g_{0}}$, then, denoting by $Y_{g}$ the generator of the flow $T_{g}$, we have

$$
\left(H_{g}\right)_{*} X_{g_{0}}:=\frac{d}{d t} H_{g} \circ T_{g_{0}}^{t}=l_{H_{g}} Y_{g}
$$

or equivalently

$$
H_{g} T_{g_{0}}^{t} v=T_{g}^{\int_{0}^{t} l_{H_{g}}\left(T_{g_{0}}^{s} v\right) d s} H_{g} v
$$

Therefore the pull-back of the length cocycle $\ell_{g}$ along $H_{\phi}$ is given by

$$
H_{g}^{*} \ell_{g}(v, t)=\int_{0}^{t} l_{H_{g}}\left(T_{g_{0}}^{s} v\right) d s
$$

and it has as generator the function $l_{H_{g}}$ defined in 3.3. Denoting by $p$ the natural projection $S M \rightarrow M$, and by $\|\cdot\|_{g}$ the norm induced by $g$ on $T M$, from $\left\|p_{*} Y_{g}\right\|_{g}=1$ we have

$$
l_{H_{g}}(v)=\left\|p_{*}\left(H_{g}\right)_{*} X_{g_{0}}(v)\right\|_{g} .
$$

The following Proposition is elementary.

3.3.3. Proposition. If the cocycles $c_{g}$ have generators $A_{g}$, then their pullback along a family $H_{g}$ of $T_{g_{0}}$-differentiable orbit equivalences is differentiable along the flow $T_{g_{0}}$ and it is generated by $\left(A_{g} \circ H_{g}\right) l_{H_{g}}$.

3.3.4. Definition. For a curve of metrics $g_{\varepsilon}$ we define the the $i$-th variation of the cocycle $c_{g_{\varepsilon}}$ as the cocycle for the flow $T_{g_{0}}$ defined by $d^{i} / d \varepsilon^{i} H_{g_{\varepsilon}}^{*} c_{g_{\varepsilon}}$ at $\varepsilon=0$.

3.3.5. Remark. Let $u_{g}$ be the generator of a cocycle $c_{g}$ for the flow $\left(\Sigma, \sigma, \lambda_{g}\right)$. Let $\bar{H}_{g}$ be orbit equivalences sending the orbits of the flow $\left(\Sigma, \sigma, \lambda_{g_{0}}\right)$ to the orbits of the flow $\left(\Sigma, \sigma, \lambda_{g}\right)$. Assume further that $\bar{H}_{g}$ is the identity on $\Sigma$ (identifying the space $X_{g}$ supporting the flow $\left(\Sigma, \sigma, \lambda_{g}\right)$ with $X_{g}=\left\{(p, t) \in \Sigma \times \mathbb{R} \mid 0 \leq t<\lambda_{g}\right\}$ and $\Sigma$ with $\left.\Sigma \times\{0\}\right)$. Then the pull-back of the length cocycle for $\left(\Sigma, \sigma, \lambda_{g}\right)$ along the orbit equivalences $\bar{H}_{g}$ has a generator $l_{g}$ satisfying

$$
\int_{0}^{\lambda_{g_{0}}} l_{g}(p, t) d t=\lambda_{g}(p)
$$


and the pull-back $\bar{H}_{g}^{*} c_{g}$ is the the cocycle generated by a function $\delta^{0} u_{g}$ such that

$$
\int_{0}^{\lambda_{g_{0}}} \delta^{0} u_{g}(p, t) d t=\int_{0}^{\lambda_{g}} u_{g}(p, t) d t
$$

We conclude that the two definitions 2.5.1 and 3.3.4 of the $i$-th variation of a cocycle agree.

Now we are ready to tackle the question of the smoothness of the dependence on $g$.

Improving on previous results of [dlLMM86], Katok et al. proved:

3.3.6. Theorem ([KKPW89]). ${ }^{3}$ For sufficiently small $\beta>0$, in a neighbourhood $\mathcal{V}^{s}$ of $g_{0}$ in $\mathcal{U}^{s}$ the family of Morse correspondences normal at $g_{0}$, $g \mapsto H_{g}$, exists and has the following properties:

1. The homeomorphisms $H_{g}$ are $T_{g_{0}}$-differentiable, that is they are differentiable along the orbits of the flow $T_{g_{0}}$.

2. The map $g \rightarrow H_{g}$ is of class $C^{s-2}$ as a map from the Banach manifold $\mathcal{V}^{s}$ to the Banach manifold of $T_{g_{0}}$-differentiable $C^{\beta}$-maps of $S M$ into itself. ${ }^{4}$

3. The map $g \mapsto l_{H_{g}}$ is of class $C^{s-2}$ as a map with values in $C^{\beta}\left(S M, \mathbb{R}^{+}\right)$.

4. The topological entropy $g \mapsto \operatorname{Ent}_{t o p}(g)$ is of class $C^{s-1}$.

Furthermore Contreras showed:

3.3.7. Theorem ([Con92]). Under the same hypotheses, denoting by $E_{g}^{s}$ the stable bundle for the flow $T_{g}$ and by $u_{g}$ the generator of the Liapunov cocycle for $T_{g}$ we have:

5. the maps $g \in \mathcal{V}^{s} \rightarrow u_{g} \circ H_{g}$ and $g \in \mathcal{V}^{s} \rightarrow E_{g}^{s} \circ H_{g}$ is of class $C^{s-3}$ as a map with values respectively in $C^{\beta}(S M)$ and in the space of $C^{\beta}$ distributions on $S M$.

${ }^{3}$ The theorem proved in [KKPW89] was stated for Anosov flows and it is valid more generally for hyperbolic attractors: see also [Con92]. We quote its application to geodesic flows. The loss of one further derivative is due to the fact that the vector field defining the geodesic flow depends on the first derivatives of the metric.

${ }^{4}$ More exactly the target space is the space $C_{g_{0}}^{\beta}(S M, S M)$ of $C^{\beta}$ continuous maps $f: S M \rightarrow S M$ whose derivatives $X_{g_{0}} f$ along the orbits of the flow $T_{g_{0}}$ are $C^{\beta}$ continuous maps $S M \rightarrow T(S M)$ endowed with the norm $\|f\|_{\beta}+\left\|X_{g_{0}} f\right\|_{\beta}$. 
6. The entropy of Liouville measure $g \mapsto \operatorname{Ent}_{\text {Liouv }}(g)$ as a function of $g \in \mathcal{V}^{s}$ is of class $C^{s-3}$.

3.3.8. In particular, from (2) above, if $s \geq 3$ the derivative of the $\left(g_{0}, g\right)$ Morse correspondence $H_{g}$ normal at $g_{0}$, is a linear map from the $C^{3}$ sections $S$ of the symmetric tensor bundle $S^{2} M$ to Hölder continuous vector fields $\Xi_{g}(S)$ along $H_{g}$.

3.3.9. Definition. We call the vector field $\Xi_{g_{0}}(S)$ the infinitesimal Morse correspondence at $g_{0}$ in the direction of $S$

3.3.10. The the infinitesimal Morse correspondence $\Xi_{g_{0}}(S)$ is differentiable along the $g_{0}$-geodesics and it satisfies a differential equation given in [FF93]. Furthermore, by the definition of normal Morse correspondence, the vector field $\Xi_{g_{0}}(S)$ is everywhere perpendicular to $X_{g_{0}}$ in the natural metric on $S_{g_{0}} M$, i.e. the projection of $\Xi_{g_{0}}(S)$ at $v \in S_{g_{0}} M$ on $M$ is $g_{0}$-perpendicular to $v$ :

$$
g_{0}\left(v, p_{*} \Xi_{g_{0}}(S)(v)\right)=0
$$

3.3.11. Lemma. Let $g_{\varepsilon}$ is a $C^{1}$-curve of $C^{5}$ Riemannian negatively curved metrics on a compact manifold $M$, and let $S_{\varepsilon}=\left.\frac{d}{d \varepsilon} g_{\varepsilon}\right|_{\varepsilon=0}$.

Then the generator of the first variation of the length cocycle at $g_{\varepsilon}$ at $\varepsilon=0$ is is cohomologous to the function:

$$
v \in S M \mapsto \delta l_{0}(v)=\frac{1}{2} S_{0}^{\vee}(v)=\frac{1}{2} S_{0}(v, v),
$$

and the generator of the first variation of the Liapunov cocycle $u_{\varepsilon}$ at $\varepsilon=0$ is cohomologous to the Hölder function:

$$
v \in S M \mapsto \delta u_{0}(v)=\left.\frac{d}{d \varepsilon} u_{\varepsilon} \circ H_{g_{\varepsilon}}(v)\right|_{\varepsilon=0}+\frac{1}{2} S_{0}(v, v) u_{0}(v) .
$$

Proof. By Proposition 3.3.3, the pull-back of the $T_{g_{\varepsilon}}$-cocycle generated by $u_{\varepsilon}$ has generator $u_{\varepsilon}\left(H_{g_{\varepsilon}}\right) l_{\varepsilon}$ and by (1)-(6) above the derivatives $d u_{\varepsilon} \circ H_{g_{\varepsilon}} / d \varepsilon$ and $d l_{\varepsilon} / d \varepsilon$ both exist as limits in $C^{\beta}(S M)$. Thus for each $g_{0}$-geodesic closed orbit $\gamma_{0}$, denoting by $\gamma_{\varepsilon}$ the geodesic $g_{\varepsilon}$-geodesic closed orbit homotopic to 
$\gamma_{0}$ we have

$$
\begin{gathered}
\frac{d}{d \varepsilon} \int_{\gamma_{\varepsilon}} u_{\varepsilon}\left(T_{g_{\varepsilon}}^{s} v\right) d s \\
=\frac{d}{d \varepsilon} \int_{\gamma_{0}} u_{\varepsilon}\left(H_{g_{\varepsilon}} T_{g_{0}}^{s} v\right) l_{H_{g_{\varepsilon}}}\left(T_{g_{0}}^{s} v\right) d s \\
=\int_{\gamma_{0}}\left[\frac{d}{d \varepsilon}\left(u_{\varepsilon}\left(H_{g_{\varepsilon}} T_{g_{0}}^{s} v\right)\right) l_{H_{g_{\varepsilon}}}\left(T_{g_{0}}^{s} v\right)+u_{\varepsilon}\left(H_{g_{\varepsilon}} T_{g_{0}}^{s} v\right) \frac{d}{d \varepsilon} l_{H_{g_{\varepsilon}}}\left(T_{g_{0}}^{s} v\right)\right] d s
\end{gathered}
$$

At $\varepsilon=0$, we have $l_{H_{\varepsilon}}\left(T_{g_{0}}^{s} v\right)=1$ and, using 3.4 and 3.5 , we see from the equation of the geodesics (cf. [FF93] that

$$
\left.\frac{d}{d \varepsilon} l_{H_{g_{\varepsilon}}}(w)\right|_{\varepsilon=0}=\frac{1}{2} S(w, w)
$$

Since, by a well known theorem of Livšic [Liv71], the collection of integrals along periodic orbits determine the cohomology class of Hölder cocycles, our claim is proved.

\section{$\S 4$. Relation of the Liapunov cocycle to the variation of metric.}

In this section we find a formula for the first variation of the Liapunov cocycle in terms of the first order variation of the Riemannian metric.

\section{1.}

Throughout this section, $M$ denotes a compact connected manifold with no boundary. We denote by $S M$ the bundle of oriented directions on $M$. As usual given a Riemannian metric $g$ of class $C^{s}$ on $M$ we identify $S M$ with the unit tangent bundle $S_{g} M=\{v \in T M \mid g(v, v)=1\}$, via the obvious $C^{s}$-diffeomorphism. The symbol $g^{t}$ denotes both the geodesic flow on $S_{g} M$ determined by $g$ can and the flow induced on $S M$, via the above identification.

We shall consider a $C^{1}$ path $\varepsilon \in\left(-\varepsilon_{0}, \varepsilon_{0}\right) \mapsto g_{\varepsilon}$ of $C^{s}$-metrics, with $s \geq 4$ and set $S_{\varepsilon}=\frac{d g_{\varepsilon}}{d \varepsilon}$. We denote by $H_{\varepsilon}: S M \rightarrow S M$ the $\left(g_{0}, g_{\varepsilon}\right)$-Morse correspondence normal at $g_{0}$ and denote by $\Xi_{g_{0}}\left(S_{g_{0}}\right)$ the infinitesimal Morse correspondence. 
As usual let $p: S M \rightarrow M$ be the canonical projection. Let $\gamma_{0}(t)$ be a unit-speed geodesic of initial velocity $\gamma_{0}^{\prime}(0)=v \in S M$. Clearly we have $\gamma_{0}(t)=p\left(g_{0}^{t} v\right)$. Define

$$
\bar{\gamma}: D=\mathbb{R} \times\left(-\varepsilon_{0}, \varepsilon_{0}\right) \mapsto \bar{\gamma}(t, \varepsilon)=p\left(h_{\varepsilon}\left(g_{0}^{t} v\right)\right) \in M .
$$

We set, for all $t, \xi=\left.\frac{\partial \bar{\gamma}}{\partial \varepsilon}\right|_{\varepsilon}=0, Y=\left.\frac{\partial \bar{\gamma}}{\partial t}\right|_{\varepsilon}=0$. Of course $Y(t)=g_{0}^{t} v$ and $\xi(t)=p_{*}\left(\Xi_{g_{0}}\left(S_{g_{0}}\right)\left(g_{0}^{t} v\right)\right)$.

The map $\bar{\gamma}$ satisfies the following conditions:

1. The map $\bar{\gamma}$ is differentiable, by Theorem 3.3.6 and our smoothness hypotheses.

2. The curves $\gamma_{\varepsilon}: t \mapsto \bar{\gamma}(t, \varepsilon)$ are geodesics for the metric $g_{\varepsilon}$ on $M$, by definition of Morse correspondence.

3. We have $g_{0}(\xi, Y)=0$, since we are considering the Morse correspondence normal at $g_{0}$.

4. We have also $g_{0}(Y, Y)=1$, since $\gamma_{0}(t)$ is a unit-speed geodesic.

Let $E$ be the pullback to $D$ of the tangent bundle of $M$ via the map $\bar{\gamma}: D \rightarrow M$. Then the bundle $E$ is endowed with Riemannian metrics $g^{\varepsilon}$ on $E$, Levi-Civita connections $\nabla^{\varepsilon}$ for the metrics $g^{\varepsilon}$, and we let $R^{\varepsilon}$ denote curvature of $\nabla^{\varepsilon}$.

Let $U$ be the section of $E^{*} \otimes E$, i.e. the $\left(\begin{array}{l}1 \\ 1\end{array}\right)$-tensor field on $M$ along $\bar{\gamma}$, defined as $U(t, \varepsilon)=U_{g_{\varepsilon}}\left(h_{\varepsilon}\left(g_{0}^{t} v\right)\right)$, where, as usual $U_{g}(v)$ is the second fundamental form of the stable horosphere of the metric $g$ at $p(v)$ considered as tensor of type $\left(\begin{array}{l}1 \\ 1\end{array}\right)$. By Theorem 3.3.7, the section $U$ is $C^{1}$.

Finally to lighten the notation we write $g, g^{t}, S, \gamma, \nabla, R$, etc. for $g_{0}, g_{0}^{t}$, $S_{0}, \gamma_{0}, \nabla^{0}, R^{0}$, etc.

4.2.1. Lemma. Denoting by $u_{\varepsilon}$ the generator of the Liapunov cocycle for that flow $g_{\varepsilon}^{t}$, along the geodesic $\gamma(t)=\gamma_{0}(t)$, we have

$$
\left.\frac{d}{d \varepsilon} u_{\varepsilon} \circ H_{g_{\varepsilon}}\left(g^{t} v\right)\right|_{\varepsilon=0}=L_{\xi} \operatorname{Tr} U(\gamma(t))=\operatorname{Tr} \nabla_{\xi} U(\gamma(t))
$$

Proof. The first equality follows from (3.2) and the definition of $\xi$. The second follows from the fact that taking traces of endomorphisms commutes with covariant differentiation. 
4.2.2. Notation. Set for simplicity

$$
B(v)=\left.\frac{d}{d \varepsilon} u_{\varepsilon} \circ H_{g_{\varepsilon}}\left(g^{t} v\right)\right|_{\varepsilon=0} .
$$

Recall that the first variation of the Liapunov cocycle along the path $g_{\varepsilon}$ at $\varepsilon=0$ is given by

$$
\delta u(v)=B(v)+\frac{1}{2} S(v, v) \operatorname{Tr} U .
$$

4.2.3. Lemma. Along the geodesic $\gamma(t)$, the field of endomorphisms $\nabla_{\xi} U(\gamma(t))$ satisfies the following differential equation:

$$
\begin{gathered}
\nabla_{Y}\left(\nabla_{\xi} U\right)+U \circ \nabla_{\xi} U+\nabla_{\xi} U \circ U= \\
-\frac{1}{2} S(Y, Y) \nabla_{Y} U-[\Gamma(Y), U]-[R(\xi, Y), U]-S(Y, Y) U^{2}+ \\
\left.\frac{\partial R_{\gamma(t)}^{\varepsilon}}{\partial \varepsilon}\right|_{\varepsilon=0}(Y, \cdot) Y+\nabla_{\xi} R(Y, \cdot) Y+R\left(\nabla_{Y} \xi, \cdot\right) Y+R(Y, \cdot) \nabla_{Y} \xi .
\end{gathered}
$$

where $[\cdot, \cdot]$ denotes the ordinary commutator of endomorphisms and $\Gamma$ denotes the $\left(\begin{array}{l}1 \\ 2\end{array}\right)$-tensor field along $\gamma$ given by $\Gamma=\left.\frac{d \nabla^{\varepsilon}}{d \varepsilon}\right|_{\varepsilon=0}$. For simplicity we have written $\Gamma(Y)$ for the contraction $\left.\frac{d \nabla_{Y}^{\varepsilon}}{d \varepsilon}\right|_{\varepsilon=0}$.

Proof. The field of endomorphisms $U$ satisfies, along each geodesic $\gamma_{\varepsilon}$, the Riccati equation 3.1 suitably corrected to take into account the fact that $t \mapsto \gamma_{\varepsilon}(t)$ is not a $g_{\varepsilon}$-unit speed geodesic. Thus:

$$
\left\|\frac{\partial \bar{\gamma}}{\partial t}\right\|_{\varepsilon} \frac{\nabla^{\varepsilon}}{\partial t} U+\left\|\frac{\partial \bar{\gamma}}{\partial t}\right\|_{\varepsilon}^{2} U^{2}=R^{\varepsilon}\left(\frac{\partial \bar{\gamma}}{\partial t}, \cdot\right) \frac{\partial \bar{\gamma}}{\partial t}
$$

where $R^{\varepsilon}$ denotes the Riemann curvature tensor of the metric $g^{\varepsilon}$.

Observe that we have:

$$
\begin{gathered}
\left.L_{\frac{\partial}{\partial \varepsilon}}\left(\left\|\frac{\partial \bar{\gamma}}{\partial t}\right\|_{\varepsilon}^{2}\right)\right|_{\varepsilon=0}= \\
\left.\frac{d}{d \varepsilon}\left(g_{\bar{\gamma}(t, \varepsilon)}^{\varepsilon}\left(\frac{\partial \bar{\gamma}}{\partial t} \bar{\gamma}_{(t, \varepsilon)}, \frac{\partial \bar{\gamma}}{\partial t} \bar{\gamma}(t, \varepsilon)^{2}\right)\right)\right|_{\varepsilon=0}= \\
S(Y, Y)+2 g\left(\nabla_{\xi} Y, Y\right)= \\
S(Y, Y)+2 g\left(\nabla_{Y} \xi, Y\right)=S(Y, Y) .
\end{gathered}
$$


where in the last equality we have used the fact that $\nabla_{Y} \xi$ and $Y$ are $g$ perpendicular.

Similarly one has

$$
\left.L_{\frac{\partial}{\partial \varepsilon}}\left(\left.\left\|\frac{\partial \bar{\gamma}}{\partial t}\right\|\right|_{\varepsilon}\right)\right|_{\varepsilon=0}=\frac{1}{2} S(Y, Y)
$$

since $\|Y\|_{0}=1$.

Furthermore:

$$
\begin{gathered}
\left.\nabla_{\frac{\partial}{\partial \varepsilon}}\left(\nabla_{\frac{\partial}{\partial t}}^{\varepsilon} U_{\bar{\gamma}(t, \varepsilon)}\right)\right|_{\varepsilon=0}=\Gamma(Y) U_{\bar{\gamma}(t, 0)}-U_{\bar{\gamma}(t, 0)} \Gamma(Y)+\left.\nabla_{\frac{\partial}{\partial \varepsilon}}\left(\nabla_{\frac{\partial}{\partial t}} U_{\bar{\gamma}(t, \varepsilon)}\right)\right|_{\varepsilon=0}= \\
\Gamma(Y) U_{\bar{\gamma}(t, 0)}-U_{\bar{\gamma}(t, 0)} \Gamma(Y)+\nabla_{Y}\left(\nabla_{\xi} U_{\bar{\gamma}(t, 0)}\right)+R(\xi, Y) U_{\bar{\gamma}(t, 0)}-U_{\bar{\gamma}(t, 0)} R(\xi, Y)
\end{gathered}
$$

where, in the last equality, we have used the fact that for commuting vector fields $X, Z$ and $\left(\begin{array}{l}1 \\ 1\end{array}\right)$ tensor fields $W$ one has:

$$
\left[\nabla_{X}, \nabla_{Z}\right] W=R(X, Z) \circ W-W \circ R(X, Z) .
$$

Finally observe that

$$
\begin{gathered}
\left.\nabla_{\frac{\partial}{\partial \varepsilon}}\left(R^{\varepsilon}\left(\frac{\partial \bar{\gamma}}{\partial t}, \cdot\right) \frac{\partial \bar{\gamma}}{\partial t}\right)\right|_{\varepsilon=0}= \\
\left.\frac{\partial R_{\gamma(t, 0)}^{\varepsilon}}{\partial \varepsilon}\right|_{\varepsilon=0}(Y, \cdot) Y+\left.\nabla_{\frac{\partial}{\partial \varepsilon}}\left(R\left(\frac{\partial \bar{\gamma}}{\partial t}, \cdot\right) \frac{\partial \bar{\gamma}}{\partial t}\right)\right|_{\varepsilon=0}= \\
\left.\frac{\partial R_{\gamma(t, 0)}^{\varepsilon}}{\partial \varepsilon}\right|_{\varepsilon=0}(Y, \cdot) Y+\nabla_{\xi} R(Y, \cdot) Y+R\left(\nabla_{\xi} Y, \cdot\right) Y+R(Y, \cdot) \nabla_{\xi} Y= \\
\left.\frac{\partial R_{\gamma(t, 0)}^{\varepsilon}}{\partial \varepsilon}\right|_{\varepsilon=0}(Y, \cdot) Y+\nabla_{\xi} R(Y, \cdot) Y+R\left(\nabla_{Y} \xi, \cdot\right) Y+R(Y, \cdot) \nabla_{Y} \xi .
\end{gathered}
$$

Taking the covariant derivative of equation (4.1) along $\xi$ and using the above observations (4.2)-(4.5) we obtain that $\nabla_{\xi} U$ satisfies the given differential equation along the geodesic $\gamma$.

\section{3.}

Assume that $(M, g)$ is a rank-1 locally symmetric space of dimension $n$. Then, we can find parallel orthonormal vector fields $Y_{1}=Y, Y_{2}, \ldots, Y_{n}$ 
along the geodesic $\gamma$ satisfying $R\left(Y, Y_{i}\right) Y=\lambda_{i}^{2} Y_{i}$, with $\lambda_{1}=0, \lambda_{j}=2$ for $1<j \leq r$, and $\lambda_{j}=1$ for $r<j \leq n$. Here $r=1,2,4$ or 8 depending on the type of symmetric space.

Furthermore, from the definition of $U$, along the geodesic $\gamma$, we have $U(\cdot)=-\sqrt{R(Y, \cdot) Y}$, i.e. $U Y_{j}=-\lambda_{j} Y_{j}$.

4.3.1. Lemma. Retaining the above notation, define $B_{j}=\left\langle\left(\nabla_{\xi} U\right) Y_{j}, Y_{j}\right\rangle$, $j=1, \ldots, n$, where $\langle\cdot, \cdot\rangle$ denotes the $g$-inner-product. Then, along the geodesic $\gamma$, we have that the $B_{j}$ satisfy the differential equation

$$
L_{Y} B_{j}-2 \lambda_{j} B_{j}=-S(Y, Y) \lambda_{j}^{2}+\left\langle\left.\frac{\partial R^{\varepsilon}}{\partial \varepsilon}\right|_{\varepsilon=0}\left(Y, Y_{j}\right) Y, Y_{j}\right\rangle
$$

Proof. Since $U$ is symmetric, we have:

$$
\begin{gathered}
\left\langle\left(U \circ \nabla_{\xi} U+\nabla_{\xi} U \circ U\right) Y_{j}, Y_{j}\right\rangle= \\
\left\langle\left(\nabla_{\xi} U\right) Y_{j}, U Y_{j}\right\rangle+\left\langle\left(\nabla_{\xi} U\right) U Y_{j}, Y_{j}\right\rangle=-2 \lambda_{j} B_{j} .
\end{gathered}
$$

Also:

$$
\begin{gathered}
\left\langle[\Gamma(Y), U] Y_{j}, Y_{j}\right\rangle= \\
\left\langle\Gamma(Y) U Y_{j}, Y_{j}\right\rangle-\left\langle U \Gamma(Y) Y_{j}, Y_{j}\right\rangle=\left\langle\Gamma(Y) U Y_{j}, Y_{j}\right\rangle-\left\langle\Gamma(Y) Y_{j}, U Y_{j}\right\rangle=0 .
\end{gathered}
$$

Similarly,

$$
\left\langle[R(\xi, Y), U] Y_{j}, Y_{j}\right\rangle=0 .
$$

From $\left\langle\nabla_{Y} \xi, Y\right\rangle=0$, and since we can also assume $R\left(Y_{j}, Y\right) Y_{j}=\lambda Y$, we have:

$$
\begin{gathered}
\left\langle R\left(\nabla_{Y} \xi, Y_{j}\right) Y, Y_{j}\right\rangle= \\
\left\langle R\left(Y, Y_{j}\right)\left(\nabla_{Y} \xi\right), Y_{j}\right\rangle=\left\langle\nabla_{Y} \xi, R\left(Y_{j}, Y\right) Y_{j}\right\rangle=\lambda\left\langle\nabla_{Y} \xi, Y\right\rangle=0 .
\end{gathered}
$$

Similarly

$$
\left\langle R\left(Y, Y_{j}\right) \nabla_{Y} \xi, Y_{j}\right\rangle=0 .
$$

Evaluating on $Y_{j}$ the right hand-side of the equation of Lemma 4.2.3 and taking the inner product of with $Y_{j}$, after using 4.6-4.10 and noticing that

$$
\nabla_{Y} U=0, \quad \nabla R=0, \quad \text { and } \quad\left\langle U^{2} Y_{j}, Y_{j}\right\rangle=\lambda_{j}^{2},
$$

we obtain the given equation for $B_{j}$. 
4.3.2. Corollary. If $(M, g)$ has constant negative curvature -1 and dimension $n, \nabla_{\xi} \operatorname{Tr} U$, along $\gamma$, satisfies the following equation:

$$
L_{Y}\left(\nabla_{\xi} \operatorname{Tr} U\right)-2\left(\nabla_{\xi} \operatorname{Tr} U\right)=(1-n) S(Y, Y)-\left.\frac{\partial \operatorname{Ric}^{\varepsilon}}{\partial \varepsilon}\right|_{\varepsilon=0}(Y, Y) .
$$

Proof.

Clearly $\sum_{j} B_{j}=\sum_{j}\left\langle\left(\nabla_{\xi} U\right) Y_{j}, Y_{j}\right\rangle=\nabla_{\xi} \operatorname{Tr} U$. From

$$
\operatorname{Ric}(X, Z)=\operatorname{Tr}(W \mapsto R(X, W) Z),
$$

we have $\sum_{j}\left\langle\left.\frac{\partial R^{\varepsilon}}{\partial \varepsilon}\right|_{\varepsilon=0}\left(Y, Y_{j}\right) Y, Y_{j}\right\rangle=\left.\frac{\partial \operatorname{Ric}^{\varepsilon}}{\partial \varepsilon}\right|_{\varepsilon=0}$. Summing over $j$ the equation of Lemma 4.3.1, since $\lambda_{j}=1$ we obtain our claim.

\section{4 .}

Let $\nabla^{*} \nabla$ denote the rough Laplacian for the metric $g=g_{0}$ on $M$. Recall that the Lichnerowicz Laplacian for a metric $g$ is defined as [Bes87, 1.180b]:

$$
\Delta_{L} S=\nabla^{*} \nabla S+\operatorname{Ric} \circ S+S \circ \operatorname{Ric}-2 R^{\circ}(S),
$$

where $\circ$ denotes the contraction symmetric tensors of rank 2 identified with $\left(\begin{array}{l}1 \\ 1\end{array}\right)$ tensor via $g$ and $R^{\circ}(S)$ is defined by ${ }^{5}$

$$
R^{\circ}(S)(Y, Z)=\operatorname{Tr}_{g} S(R(\cdot, X) Y, \cdot) .
$$

Then [Bes87, 1.174]:

$$
\left.\frac{\partial \mathrm{Ric}^{\varepsilon}}{\partial \varepsilon}\right|_{\varepsilon=0}=\frac{1}{2} \Delta_{L} S-\delta^{*} \delta S-\nabla d(\operatorname{Tr} S)
$$

where $\delta^{*}$ is the symmetrization of covariant derivative and $\delta$ is its formal adjoint, the divergence.

Proof of Proposition 1.3.3. Form Corollary 4.3.2 we obtain that along the geodesic $\gamma$ we have

$$
B\left(g^{t} v\right) \nabla_{\xi}=\operatorname{Tr} U(\gamma(t)) \approx \frac{n-1}{2} S(Y(t), Y(t))+\left.\frac{1}{2} \frac{\partial \operatorname{Ric}^{\varepsilon}}{\partial \varepsilon}\right|_{\varepsilon=0}(Y(t), Y(t)),
$$

${ }^{5}$ Our definition agrees with [Bes87, 1.131], in spite of the opposite convention on the sign of the Riemann curvature $R$. 
with $\approx$ meaning that the difference is a coboundary. In fact, the difference at $\gamma(t)=p\left(g^{t} v\right)$ is $\frac{1}{2} L_{Y}\left(\nabla_{\xi} \operatorname{Tr} U\right)(\gamma(t))=\frac{1}{2} \frac{\partial}{\partial t} B\left(g^{t} v\right)$

We claim that since $g$ has constant curvature -1 we have:

$$
\Delta_{L} S(Y, Y)=\nabla^{*} \nabla S(Y, Y)-2 n S(Y, Y)+2 \operatorname{Tr}_{g} S .
$$

In fact, Ric $=-(n-1) g$, and therefore

$$
\operatorname{Ric} \circ S+S \circ \text { Ric }=-2(n-1) S .
$$

Furthermore, since $R(Y, X) Z=-\langle X, Z\rangle Y+\langle Y, Z\rangle X$

$$
\begin{aligned}
R^{\circ}(S)(Y, Y)= & \sum_{i} S\left(R\left(Y_{i}, Y\right) Y, Y_{i}\right)= \\
& =\sum_{i} S\left(-Y_{i}+\left\langle Y, Y_{i}\right\rangle Y, Y_{i}\right)=-\operatorname{Tr}_{g}(S)+S(Y, Y) .
\end{aligned}
$$

proving our claim. We obtain that

$$
\begin{aligned}
\nabla_{\xi} \operatorname{Tr} U \approx & \frac{n-1}{2} S(Y, Y)+\frac{1}{4} \nabla^{*} \nabla S(Y, Y)-\frac{n}{2} S(Y, Y)+ \\
& +\frac{1}{2} \operatorname{Tr}_{g} S-\frac{1}{2} \delta^{*} \delta S(Y, Y)-\frac{1}{2} \nabla d(\operatorname{Tr} S)(Y, Y) \\
\approx- & \frac{1}{2} S(Y, Y)+\frac{1}{4} \nabla^{*} \nabla S(Y, Y)+\frac{1}{2} \operatorname{Tr}_{g} S- \\
& -\frac{1}{2} \delta^{*} \delta S(Y, Y)-\frac{1}{2} \nabla d(\operatorname{Tr} S)(Y, Y) .
\end{aligned}
$$

and setting

$$
T=-\frac{1}{2} S+\frac{1}{4} \nabla^{*} \nabla S+\frac{1}{2}\left(\operatorname{Tr}_{g} S\right) g-\frac{1}{2} \delta^{*} \delta S
$$

and noticing that the term $\nabla d(\operatorname{Tr} S)(Y, Y)=Y^{2}(\operatorname{Tr} S)$ is cohomologous to zero, we have concluded that

$$
B\left(g^{t} v\right)=\nabla_{\xi} \operatorname{Tr} U(\gamma(t)) \approx T^{\vee}\left(g^{t} v\right)
$$

or $B \approx T^{\vee}$. Thus the first variation of the Liapunov cocycle along the path $g_{\varepsilon}$ at $\varepsilon=0$ is

$$
\delta u=B+\frac{1}{2} S^{\vee} \operatorname{Tr} U \approx T^{\vee}-\frac{n-1}{2} S^{\vee} .
$$




\section{§5. Proof of Proposition 1.3.4 and Theorem C.}

\section{1 .}

Let $g_{\varepsilon} \in \mathcal{U}$ be a curve of metrics of constant volume on $M$. Then if we set $S_{\varepsilon}=\frac{\partial g_{\varepsilon}}{\partial \varepsilon}$ and denoting by $s_{n}$ the volume of the $n$-sphere, we have,

$$
\begin{aligned}
& \frac{1}{2} \int_{S g_{\varepsilon} M} S^{\vee}(v) d \operatorname{Liouv}\left(g_{\varepsilon}\right)(v)= \\
&=\frac{s_{n-1}}{2 n} \int_{M} \operatorname{Tr}_{g_{\varepsilon}} S_{\varepsilon} d \operatorname{Vol} g_{\varepsilon}=-\frac{s_{n-1}}{n} \frac{\partial \operatorname{Vol}_{g_{\varepsilon}}(M)}{\partial \varepsilon}=0 .
\end{aligned}
$$

Since $\frac{1}{2} S^{\vee}$ is the first variation $\delta l_{\varepsilon}$ of the length cocycle $l_{\varepsilon}$, and $\operatorname{Liouv}\left(g_{\varepsilon}\right)$ the Gibbs state for the Liapunov cocycle $u_{\varepsilon}$ of the flow $T_{g_{\varepsilon}}$, we obtain that $m_{u_{\varepsilon}}\left(\delta l_{\varepsilon}\right)=0$ for all $\varepsilon$. If $g_{0}$ is locally symmetric, the measure of maximal entropy $M_{0}$ and the Liouville measure $\operatorname{Liouv}\left(g_{0}\right)$ coincide and thus all the conditions of Proposition 1.3.1 (C) are verified. Denoting for simplicity $g_{0}$ by $g$, the maximal measure entropy $M_{0}$ by $m$ and the covariance of the flow $T_{g}$ with respect to the measure of $m$ by Cov, we can summarize the results so far achieved (cf. Propositions 1.3.1, 1.3.3) in the following Proposition:

5.1.1. Proposition. Let $g_{\varepsilon}$ be a $C^{2}$-curve of $C^{5}$-metrics on a manifold $M$ of constant volume, and assume that that $g=g_{0}$ has constant negative curvature -1. Denoting with $\delta l$ and $\delta u$ the first variation of the length cocycle and of Liapunov cocycle at $\varepsilon=0$ we have:

$$
\operatorname{Ent}_{\text {top }}^{\prime \prime}(g)=\operatorname{Cov}((n-1) \delta l+\delta u,(n-1) \delta l)
$$

and

$$
\operatorname{Ent}_{\text {Liouv }}^{\prime \prime}(g)=-\operatorname{Cov}((n-1) \delta l+\delta u, \delta u) .
$$

Setting $T=\mathcal{T}(S):=-\frac{1}{2} S+\frac{1}{4} \nabla^{*} \nabla S+\frac{1}{2}\left(\operatorname{Tr}_{g} S\right) g-\frac{1}{2} \delta^{*} \delta S$, since $\delta l \approx \frac{1}{2} S^{\vee}$ and $\delta u \approx T^{\vee}-\frac{n-1}{2} S^{\vee}$, we have

$$
\begin{gathered}
\operatorname{Ent}_{\text {top }}^{\prime \prime}(g)=\operatorname{Cov}\left(T^{\vee}, \frac{n-1}{2} S^{\vee}\right), \\
\operatorname{Ent}_{\text {Liouv }}^{\prime \prime}(g)=-\operatorname{Cov}\left(T^{\vee}, T^{\vee}-\frac{n-1}{2} S^{\vee}\right)
\end{gathered}
$$

and

$$
\operatorname{Ent}_{\text {top }}^{\prime \prime}(g)-\operatorname{Ent}_{\text {Liouv }}^{\prime \prime}(g)=\operatorname{Cov}\left(T^{\vee}, T^{\vee}\right)
$$




\section{2.}

If $g$ has constant sectional curvature, then the group $G$ of isometries of $\widetilde{M}$, the universal cover of $M$, is isomorphic to $S O_{0}(1, n)$, where $n=$ $\operatorname{dim}(M)$. We have an identification of $M$ with the space $\Gamma \backslash G / K$, where $\Gamma \approx \pi_{1}(M)$ is a discrete group of isometries acting without fixed points on $\widetilde{M}$, and $K \approx S O(n)$ is the stabilizer of a point $p_{0} \in \widetilde{M}$. Furthermore, the unit tangent unit bundle $S_{g} M$ and the orthonormal frame bundle $F M$ of $M$ are identified with $\Gamma \backslash G / K_{1}$ and $\Gamma \backslash G$, where $K_{1} \approx S O(n-1)$ is the stabilizer of a vector $v_{0} \in T_{p_{0}} \widetilde{M}$. The parallel transport of an orthonormal frame $\left(v_{1}, v_{2}, \ldots, v_{n}\right) \in F M$ along the geodesic determined by $v_{1}$ is identified with the action on $\Gamma \backslash G$ given by multiplication on the right by the splitCartan $A_{1} \approx \mathbb{R}$ commuting with $K_{1}$. It is plain that this parallel transport projects via the natural projection $\left(v_{1}, v_{2}, \ldots, v_{n}\right) \in F M \mapsto v_{1} \in S_{g} M$ to the geodesic flow on $S_{g} M$.

5.2.1. Notation. Denote by $\mathcal{C}^{r}\left(S^{2} M\right)$ the space of $C^{r}$-sections of the bundle $S^{2} M$ of symmetric covariant tensors of rank 2. Similarly $\mathcal{L}^{2}\left(S^{2} M\right)$ denotes the $L^{2}$ sections of this bundle. Clearly the map ${ }^{\vee}: S \rightarrow S^{\vee}$ defined, for $v \in S_{g} M$, by $S^{\vee}(v)=S(v, v)$ maps $\mathcal{C}^{r}\left(S^{2} M\right)$ to $C^{r}\left(S_{g} M\right)$ and $\mathcal{L}^{2}\left(S^{2} M\right)$ to $L^{2}\left(S_{g} M\right)$.

Observe also that we have an injection $L^{2}\left(S_{g} M\right) \hookrightarrow L^{2}(F M)$, regarding $L^{2}\left(S_{g} M\right)$ as the subset of $L^{2}(F M)$ of $K_{1}$-invariant vectors. Similarly, $C^{r}\left(S_{g} M\right) \hookrightarrow C^{r}(F M)$. In the sequel these identifications will be implicit.

5.2.2. Left invariant differential operators on $G$ commuting with $K_{1}$ act on $C^{\infty}\left(S_{g} M\right)$. This is in particular true of the the Casimir operator $\operatorname{Cas}_{S O(1, n)}$ of $G$, which we normalize so that on $C^{\infty}(M)$ it coincides with the rough Laplacian $\nabla^{*} \nabla$.

Observe that the differential operators $\nabla^{*} \nabla, \delta, \delta^{*}$ etc. acting on symmetric tensor field can be viewed by means of the above maps as left invariant differential operators on $C^{\infty}(F M)$ commuting with $K_{1}$. In fact, denote by $P_{i}$ the parallel transport of an orthonormal frame $\left(v_{1}, v_{2}, \ldots, v_{n}\right) \in F M$ along the geodesic determined by $v_{i}$ and let $Y_{i}$ be vector field generating the flow $P_{i}$. For $i<j$, set $R_{i j}=\left[Y_{i}, Y_{j}\right]$. Then it is plain that the vector fields $Y_{i}, R_{i j},(i<j)$, form a basis for the Lie algebra $\mathfrak{g} \approx \mathfrak{s o}(1, n)$ of $G$ and that $R_{i j}$ exponentiate to the flow $\exp \left(\theta R_{i j}\right)$ which rotates an orthonormal frame $\left(v_{1}, \ldots, v_{i}, \ldots, v_{j}, \ldots, v_{n}\right) \in F M$ to $\left(v_{1}, \ldots, \cos (\theta) v_{i}-\right.$ $\left.\sin (\theta) v_{j}, \ldots, \sin (\theta) v_{i}+\cos (\theta) v_{j}, \ldots, v_{n}\right)$. In particular given $S \in \mathcal{C}^{\in}\left(\mathcal{S}^{\in \mathcal{M}}\right)$, 
setting, for $\left(v_{1}, \ldots, v_{n}\right) \in F M$,

$$
S_{i j}\left(v_{1}, \ldots, v_{n}\right)=S\left(v_{i}, v_{j}\right)
$$

and identifying $S_{11}$ with the lift to $F M$ of $S^{\vee}$ we have

$$
S_{1 i}=-\frac{1}{2} L_{R_{1 i}} S^{\vee}
$$

Thus, if $\left(v_{1}, \ldots, v_{i}, \ldots, v_{j}, \ldots, v_{n}\right) \in F M$ and $S \in C^{2}\left(S^{2} M\right)$, we have:

$$
\begin{aligned}
(\delta S)^{\vee}\left(v_{1}\right) & =(\delta S)\left(v_{1}\right)=-\sum_{i} \nabla_{v_{i}} S\left(v_{i}, v_{1}\right)=-\left.\sum_{i} \frac{\partial}{\partial t} S\left(P_{i}^{t} v_{i}, P_{i}^{t} v_{1}\right)\right|_{t=0} \\
& =-\left.\sum_{i} S_{1 i}\left(P_{i}^{t} v_{1}, \ldots, P_{i}^{t} v_{n}\right)\right|_{t=0}=-\sum_{i} L_{Y_{i}} S_{1 i}\left(v_{1}, \ldots, v_{n}\right) \\
& =\frac{1}{2} \sum_{i} L_{Y_{i}} L_{R_{1 i}} S^{\vee}\left(v_{1}, \ldots, v_{n}\right)
\end{aligned}
$$

Similarly, we have

$$
\nabla^{*} \nabla=-\sum_{1} L_{Y_{i}}^{2}=\left(-\sum_{i} L_{Y_{i}}^{2}+\sum_{i<j} R_{i j}^{2}\right)-\sum_{i<j} R_{i j}^{2}=\operatorname{Cas}_{S O(1, n)}-\operatorname{Cas}_{S O(n)}
$$

where $\operatorname{Cas}_{G}$ denotes the Casimir operator of the group $G$. Since, for $S \in \mathcal{C}^{\in}\left(\mathcal{S}^{\in} \mathcal{M}\right)$, we have $\operatorname{Cas}_{S O(n)} S^{\vee}=2 \operatorname{Tr} S-2 n S^{\vee}$, we can express the operator $\mathcal{T}(\mathcal{S})$ defined in 5.1.1 as

$$
\begin{aligned}
\mathcal{T}(\mathcal{S}) & =-\frac{1}{2} S+\frac{1}{4} \nabla^{*} \nabla S+\frac{1}{2}\left(\operatorname{Tr}_{g} S\right) \otimes g-\frac{1}{2} \delta^{*} \delta S \\
& =-\frac{1}{2} S+\frac{1}{4}\left(\operatorname{Cas}_{S O(1, n)}-\mathbf{C a s}_{S O(n)}\right) S+\frac{1}{2}\left(\operatorname{Tr}_{g} S\right) \otimes g-\frac{1}{2} \delta^{*} \delta S \\
& =\frac{n-1}{2} S+\frac{1}{4} \mathbf{C a s}_{S O(1, n)} S-\frac{1}{2} \delta^{*} \delta S .
\end{aligned}
$$

\section{3.}

By a well known theorem on the unitary representation of $S O_{0}(1, n)$, the Hilbert space $L^{2}(F M) \approx \Gamma \backslash G$ splits as a direct sum $L^{2}(F M)=\sum H_{i}$ of topologically irreducible components $H_{i}$ of the right action of $G$. 
5.3.1. Lemma. Each irreducible representation space $H_{i}$ splits as a direct sum of irreducible representation $H_{j, \tau} \approx \tau$ for the action of the maximal compact subgroup $K \approx S O(n)$ of $G$. Let $\tau$ be an irreducible representation of $S O(n)$. Then the multiplicity of $\tau$ in $H_{i}$ is at most one.

Proof. The first statement is trivial. Denote by $P=M A N$ a minimal parabolic of $G=S O_{0}(1, n)$. By a Theorem of Harish-Chandra [War72, 5.5.1.7], there is a irreducible representation $\sigma$ of $M \approx S O(n-1)$, such that the multiplicity of $\tau$ in $H_{i}$ is at most the multiplicity of $\sigma$ in the restriction of $\tau$ to $M$. This concludes the proof, since the multiplicity of any irreducible representations of $S O(n-1)$ in a given irreducible representation of $S O(n)$ is at most one (cf. [Žel73, §129] and [GC50]).

5.3.2. Notation. For each equivalence class of irreducible representations $\tau$ of $K$, let $H_{\tau}$ be the subspace of $H$ of $K$-type $\tau$ i.e. the set of vectors transforming under $K$ according to the irreducible representation $\tau$ of $K$.

The following lemma is an easy consequence of the ellipticity of the rough Laplacian $\nabla^{*} \nabla$ acting on symmetric tensors. For $S \in \mathcal{C}^{\infty}\left(S^{p} M\right)$, we set $S^{\vee}(v)=S(v, v, \ldots, v)$.

5.3.3. Lemma. Let $S \in \mathcal{C}^{\infty}\left(S^{p} M\right)$ be a symmetric tensor field of rank $p$ and $K$-type $\tau$. Let $S^{\vee}=\sum s_{i}, s_{i} \in L^{2}(F M)$, be its decomposition in irreducible components. Then we have

1. For each $s_{i}$ there exists a symmetric tensor field $S_{i} \in \mathcal{C}^{\infty}\left(S^{p} M\right)$ of rank $p$ and $K$-type $\tau$ with $s_{i}=S_{i}^{\vee}$.

2. Each $S_{i}$ is an eigenfunction of the rough Laplacian $\nabla^{*} \nabla$ and, in particular, $S_{i}$ is $C^{\infty}$.

3. The series $S=\sum S_{i}$, or equivalently $S^{\vee}=\sum s_{i}$, converges in the $C^{\infty}$ topology.

We will only be interested with symmetric tensor field of rank 2 and therefore with only two $K$-types: the trivial $K$-type and the $K$-type of traceless symmetric tensor of rank 2 .

5.3.4. Notation. Set

$$
\operatorname{Conf}^{r}(g)=\left\{S: S=f \otimes g, f \in C^{r}(M)\right\}
$$


and

$$
\operatorname{Teich}^{r}(g):=\left\{S \in \mathcal{C}^{r}\left(S^{2} M\right): \delta S=0, \operatorname{Tr}_{g} S=0\right\} .
$$

Then $\operatorname{Conf}^{r}(g)$ is the space tangent to $C^{r}$-conformal deformation of $g$ and it is the space of vectors of the trivial $K$-type in $C^{r}(F M)$. The space Teich ${ }^{r}(g)$ is the formal $L^{2}$-orthogonal space in $\mathcal{C}^{r}\left(S^{2} M\right)$ to the conformal fibers and to the local orbit of $g$ under the diffeomorphism group. Furthermore, each $S \in \operatorname{Teich}^{r}(g)$ has the $K$-type of traceless symmetric tensor of rank 2 .

5.3.5. Notation. Let $S \in L^{2}\left(S^{2} M\right)$. Let $H(S)$ be the cyclic subspace of $L^{2}(F M)$ generated by $G$ acting on $S^{\vee}$.

5.3.6. Corollary. Let $R \in \operatorname{Conf}^{\infty}(g)$ and write $R^{\vee}=\sum_{i} r_{i}$, with $r_{i} \in H_{i}$. Then, for all $i$, we have $r_{i}=R_{i}^{\vee}$ with $R_{i} \in \operatorname{Conf}^{\infty}(g)$.

Similarly, let $S \in \operatorname{Teich}^{\infty}(g)$ and write $S^{\vee}=\sum_{i} s_{i}$, with $s_{i} \in H_{i}$. Then, for all $i$, we have $s_{i}=S_{i}^{\vee}$ with $S_{i} \in \operatorname{Teich}^{\infty}(g)$.

Proof. The first claim is a mere restatement of Lemma 5.3.3. If $S \in$ Teich ${ }^{\infty}(g)$ and $S^{\vee}=\sum_{i} s_{i}$, by Lemma 5.3 .3 we have $s_{i}=S_{i}^{\vee}$ with $S_{i}$ smooth traceless symmetric tensor field of rank 2. The discussion in 5.2.2 implies that the divergence operator on smooth traceless symmetric tensor field of rank 2 coincides with an element of the enveloping algebra of $\mathfrak{g} \approx \mathfrak{s o}(1, n)$. Thus we have $\delta s_{i} \in H_{i}$ for all $i$. We conclude that $\delta S=0$ implies $\delta S_{i}=0$ for all $i$, showing that $S_{i} \in \operatorname{Teich}^{\infty}(g)$ for all $i$.

5.3.7. Lemma. Let $R \in \operatorname{Conf}^{\infty}(g)$ and $S \in \operatorname{Teich}^{\infty}(g)$ then $H(R) \perp H(S)$.

Proof. By Corollary 5.3.6, is sufficient to consider the case in which $R$ and $S$ belong to the same irreducible subspace $H_{i}$. Arguing by contradiction, we may assume that $H_{i}=H(R)=H(S)$. Then $R=r \otimes g$ with $r \in C^{\infty}(M)$. By the ergodicity of the geodesic flow, we have that $\delta^{*} \delta^{*} r=\nabla d r$ is not zero. In fact, for $v \in S_{g} M$ we have $\left(\delta^{*} \delta^{*} r\right)^{\vee}(v)=\nabla_{v, v}^{2} r=d^{2} /\left.d t^{2} r\left(g^{t} v\right)\right|_{t=0}$, thus $\delta^{*} \delta^{*} r=0$ would imply that $r$ is constant, in which case there is nothing to prove.

It is not difficult to see from the compactness of $M$ that the traceless part $(\nabla d r)_{0}:=\nabla d r-\frac{\Delta r}{n} \otimes g$ of $\nabla d r$ is not zero. Since $S$ is traceless, the $K$ orbits of $(\nabla d r)_{0}^{\vee}$ and $S^{\vee}$ generate $K$-representations both isomorphic to the representation of $S O(n)$ on the space of harmonic homogeneous polynomial 
of degree two on $\mathbb{R}^{n}$. By Lemma 5.3.1 the multiplicity of this representation in $H_{i}$ is 1 , and therefore $(\nabla d r)_{0}^{\vee}$ and $S^{\vee}$ have the same $K$-orbit. However, since $(\nabla d r)_{0}^{\vee}$ and $S^{\vee}$ are both $K_{1}$-invariant we obtain that, for some constant $C \neq 0$, we have $S^{\vee}=C(\nabla d r)_{0}^{\vee}$ and equivalently $S=C(\nabla d r)_{0}$. From $\left\langle S,(\nabla d r)_{0}\right\rangle=\left\langle S,\left(\delta^{*} \delta^{*} r\right)_{0}\right\rangle=\left\langle S, \delta^{*} \delta^{*} r\right\rangle$, we obtain $\left\langle S, \delta^{*} \delta^{*} r\right\rangle \neq 0$. This is impossible because we have $\left\langle S, \delta^{*} \delta^{*} r\right\rangle=\left\langle\delta S, \delta^{*} r\right\rangle$, and the latter term is zero since $S \in \operatorname{Teich}(g)$ and therefore $S$ is divergence free.

5.3.8. Remark. A simple modification of the proof above shows that if $S \in \operatorname{Teich}^{\infty}(g)$, then $H(S)$ is orthogonal to all one forms. This means that if $S \in \operatorname{Teich}^{\infty}(g)$, then the minimal $K$-type occurring in $H(S)$ is the $K$-type of $S$.

5.3.9. Lemma. Let $S_{1}, S_{2} \in \mathcal{C}^{\infty}\left(S^{p} M\right)$ and assume that $H\left(S_{1}\right)$ and $H\left(S_{2}\right)$ are orthogonal. Then

$$
\operatorname{Cov}\left(S_{1}^{\vee}, S_{2}^{\vee}\right)=\operatorname{Cov}\left(\mathcal{T}\left(S_{1}\right)^{\vee}, S_{2}^{\vee}\right)=\operatorname{Cov}\left(\mathcal{T}\left(S_{1}\right)^{\vee}, \mathcal{T}\left(S_{1}\right)^{\vee}\right)=0
$$

Proof. By formula 5.1 the operator $\mathcal{T}$ belongs to the enveloping algebra of $G$ and therefore $\mathcal{T}\left(S_{i}\right) \in H\left(S_{i}\right)$.

Since the flow $g^{t}$ (lifted to $F M$ ) is given by right translation of by some split-Cartan in $G$, by the exponential decay of matrix coefficients, we have

$$
\operatorname{Cov}(F, G)=\int_{-\infty}^{\infty}\left\langle F, G \circ g^{t}\right\rangle d t
$$

for any pair of smooth functions $F$ and $G$ on $F M$, provided that either $F$ or $G$ integrates to zero on $F M$. Thus if $F$ and $G$ belong to orthogonal $G$-invariant subspaces we have that at least one of them has average zero on $F M$ and $\operatorname{Cov}(F, G)=0$. The lemma follows.

Proof of Proposition 1.3.4. Recall that for simplicity we write $g$ instead of $g_{0}$. It is sufficient to prove the claim for a dense set of $S \in C^{3}\left(S^{2} M\right)$, e.g. for $S \in C^{\infty}\left(S^{2} M\right)$, since the bilinear form $\mathrm{Cov}$ is continuous in the $C^{\alpha}$ topology. For $S$ in $\mathcal{C}^{\infty}\left(S^{2} M\right)$ we can write

$$
S=S_{T}+S_{C}+S_{0}
$$


with $S_{T} \in$ Teich $^{\infty}(g), S_{C} \in \operatorname{Conf}^{\infty}(g)$ and with $S_{0}$ belonging to the image under $\delta^{*}$ of the space $\Lambda^{1}(M)$ of $C^{\infty}$ one-forms. This decomposition of $S$ is unique, since the sum

$$
C^{\infty}\left(S^{2} M\right)=\operatorname{Teich}^{\infty}(g)+\operatorname{Conf}^{\infty}(g)+\delta^{*}\left(\Lambda^{1}(M)\right)
$$

is direct (Cf. [Bes87, Lemma 4.57]). By the linearity of the operator $\mathcal{T}$, we can decompose $T$ as $T=\mathcal{T}\left(S_{T}\right)+\mathcal{T}\left(S_{C}\right)+\mathcal{T}\left(S_{0}\right)$. Now, we observe that, in computing $\operatorname{Cov}\left(T^{\vee}, S^{\vee}\right)$ and $\operatorname{Cov}\left(T^{\vee}, T^{\vee}\right)$, the terms $S_{0}$ and $\mathcal{T}\left(S_{0}\right)$ are irrelevant: in fact the terms $S_{0}^{\vee}$ and $\mathcal{T}\left(S_{0}\right)^{\vee}$ are cohomologous to zero, since we have $S_{0}=L_{X} g$ for some $C^{\infty}$ vector field $X$ on $M$. Thus the proof of Proposition 1.3.4 will be complete if we prove it simply for $S \in$ $\operatorname{Teich}^{\infty}(g)+\operatorname{Conf}^{\infty}(g)$.

By Lemma 5.3.9, we have

$$
\operatorname{Cov}\left(\mathcal{T}\left(S_{C}\right)^{\vee}, S_{T}^{\vee}\right)=\operatorname{Cov}\left(\mathcal{T}\left(S_{T}\right)^{\vee}, S_{C}^{\vee}\right)=\operatorname{Cov}\left(\mathcal{T}\left(S_{C}\right)^{\vee}, \mathcal{T}\left(S_{T}\right)^{\vee}\right)=0
$$

and thus it suffices to prove the claim of Proposition 1.3.4 separately for $S \in \operatorname{Teich}^{\infty}(g)$ and for $S \in \operatorname{Conf}^{\infty}(g)$.

Let $S \in \operatorname{Teich}^{\infty}(g)$. From $\mathcal{T}(S)=-\frac{1}{2} S+\frac{1}{4} \nabla^{*} \nabla S+\frac{1}{2}\left(\operatorname{Tr}_{g} S\right) g-\frac{1}{2} \delta^{*} \delta S$ and the definition of $\operatorname{Teich}^{\infty}(g)$ we obtain

$$
\mathcal{T}(S)=-\frac{1}{2} S+\frac{1}{4} \nabla^{*} \nabla S
$$

Regard $S$ as a one-form with values in $T^{*} M$. Let $d^{\nabla}$ be the differential induced on $\Lambda^{r}(M) \otimes T^{*}(M)$ by the Levi-Civita connection and let $d^{\nabla^{*}}$ be its formal adjoint. We have the following Weitzenbock formula (cf. [Bes87, p.335])

$$
\left(d^{\nabla^{*}} d^{\nabla}+d^{\nabla} d^{\nabla^{*}}\right) S=\nabla^{*} \nabla S-R^{\circ}(S)+S \circ \text { Ric } .
$$

where $R^{\circ}(S)$ was defined in (4.12). Using the formula (4.14) and the fact that Ric $=-(n-1) g$, we obtain

$$
\left(d^{\nabla^{*}} d^{\nabla}+d^{\nabla} d^{\nabla^{*}}\right) S=\nabla^{*} \nabla S-S-(n-1) S=\nabla^{*} \nabla S-n S .
$$

Since the left hand side above is positive, we conclude that the spectrum of the restriction of the $\nabla^{*} \nabla$ to $\operatorname{Teich}^{\infty}(g)$ lies in $[n, \infty)$ and (5.2) implies that the spectrum of the restriction of $\mathcal{T}(S)$ to $\operatorname{Teich}^{\infty}(g)$ lies in the interval $[(n-2) / 4, \infty)$.

By Lemma 5.3.3, we can decompose $S$ as a $C^{\infty}$ convergent series $S=\sum S_{i}$ with $S_{i}^{\vee}$ belonging to the irreducible component $H_{i}$ and $S_{i}$ eigenfunction of $\nabla^{*} \nabla$ of eigenvalue $\lambda_{i} \geq(n-2) / 4$. Then for $i \neq j$ the spaces $H\left(S_{i}\right)$ and $H\left(S_{j}\right)$ are orthogonal. 
Lemma 5.3.9 implies that

$$
\operatorname{Cov}\left(S_{i}^{\vee}, S_{j}^{\vee}\right)=\operatorname{Cov}\left(\mathcal{T}\left(S_{i}\right)^{\vee}, S_{j}^{\vee}\right)=\operatorname{Cov}\left(\mathcal{T}\left(S_{i}\right)^{\vee}, \mathcal{T}\left(S_{j}\right)^{\vee}\right)=0
$$

We conclude that

$$
\begin{aligned}
\operatorname{Cov}\left(\mathcal{T}(S)^{\vee}, S^{\vee}\right) & =\sum_{i} \operatorname{Cov}\left(\mathcal{T}\left(S_{i}\right)^{\vee}, S_{i}^{\vee}\right)=\sum_{i} \lambda_{i} \operatorname{Cov}\left(S_{i}^{\vee}, S_{i}^{\vee}\right) \\
& \geq \sum_{i} \frac{n-2}{4} \operatorname{Cov}\left(S_{i}^{\vee}, S_{i}^{\vee}\right)=\frac{n-2}{4} \operatorname{Cov}\left(S^{\vee}, S^{\vee}\right)
\end{aligned}
$$

and, similarly,

$$
\begin{array}{r}
\operatorname{Cov}\left(\mathcal{T}(S)^{\vee}, \mathcal{T}(S)^{\vee}\right)=\sum_{i} \operatorname{Cov}\left(\mathcal{T}\left(S_{i}\right)^{\vee}, \mathcal{T}\left(S_{i}\right)^{\vee}\right)=\sum_{i} \lambda_{i}^{2} \operatorname{Cov}\left(S_{i}^{\vee}, S_{i}^{\vee}\right) \\
\geq \sum_{i}\left(\frac{n-2}{4}\right)^{2} \operatorname{Cov}\left(S_{i}^{\vee}, S_{i}^{\vee}\right)=\left(\frac{n-2}{4}\right)^{2} \operatorname{Cov}\left(S^{\vee}, S^{\vee}\right)
\end{array}
$$

proving the desired estimate for the case $S \in \operatorname{Teich}^{\infty}(g)$.

Consider now the case $S \in \operatorname{Conf}^{\infty}(g)$. We have $\operatorname{Tr}_{g} S=n S$ and therefore $\mathcal{T}(S)=\frac{n-1}{2} S+\frac{1}{4} \nabla^{*} \nabla S-\frac{1}{2} \delta^{*} \delta S$. Writing $S=F \otimes g$, with $F \in C^{\infty}(M)$, we see that the term $\delta^{*} \delta S$ appearing in $\mathcal{T}(S)$ can be rewritten as $\delta d F$ and it gives rise in $\mathcal{T}(S)^{\vee}$ to the term $\left(\delta^{*} \delta S\right)^{\vee}=(\delta d F)^{\vee}$, which is cohomologous to zero. Thus, in computing $\operatorname{Cov}\left(\mathcal{T}(S)^{\vee}, S^{\vee}\right)$ and $\operatorname{Cov}\left(\mathcal{T}(S)^{\vee}, T^{\vee}\right)$ we can replace $\mathcal{T}(S)$ by

$$
\mathcal{T}_{0}(S):=\frac{n-1}{2} S+\frac{1}{4} \nabla^{*} \nabla S
$$

The positivity of the rough Laplacian $\nabla^{*} \nabla$ implies that the elliptic operator $\mathcal{T}_{0}$ has spectrum in $[(n-1) / 2, \infty)$. Reasoning as before we obtain, for $S \in \operatorname{Conf}^{\infty}(g)$, estimates similar to (5.3) and (5.4), but with the constant $(n-1) / 2$ replacing the smaller constant $(n-2) / 4$. Thus the estimates $(5.3)$ and (5.4) are also valid for $S \in \operatorname{Conf}^{\infty}(g)$. This concludes our proof

Proof of Theorem $C$. Let $n=\operatorname{dim} M=3$. By Proposition 5.1.1 and formulas (5.1) and (5.2), we see that, for $S \in \operatorname{Teich}^{\infty}(g)$,

$$
\begin{aligned}
\operatorname{Ent}_{\text {Liouv }}^{\prime \prime}(g)=-\operatorname{Cov} & \left(T^{\vee}, T^{\vee}-S^{\vee}\right)= \\
& =-\operatorname{Cov}\left(S^{\vee}+\frac{1}{4} \operatorname{Cas}_{S O(1,3)} S^{\vee}, \frac{1}{4} \operatorname{Cas}_{S O(1,3)} S^{\vee}\right) .
\end{aligned}
$$


Thus assuming that $\mathbf{C a s}_{S O(1,3)} S^{\vee}=\lambda S^{\vee}$, we obtain $\operatorname{Ent}_{\text {Liouv }}^{\prime \prime}(g)=-\frac{1}{4} \lambda(1+$ $\left.\frac{1}{4} \lambda\right) \operatorname{Cov}\left(S^{\vee}, S^{\vee}\right)$. Thus Ent'tiouv $(g)<0$ is equivalent to $\lambda>0$ or $\lambda<-4$. The latter eventuality is impossible, since we have shown that, in dimension 3 , the spectrum of the Laplacian on Teich $(g)$ is contained in the interval $[3, \infty)$ and $\operatorname{Cas}_{S O(1,3)}=\nabla^{*} \nabla+\operatorname{Cas}_{S O(3)}=\nabla^{*} \nabla-6$. In any case, since the spectrum of $\nabla^{*} \nabla$ is discrete, we find that on a infinite dimensional subspace of Teich $(g)$ the derivative $\operatorname{Ent}_{\text {Liouv }}^{\prime \prime}(g)$ in a curve of metrics in a direction in this subspace is negative.

However if the operator $\operatorname{Cas}_{S O(1,3)}$ on $\operatorname{Teich}^{\infty}(g)$ has an eigenvector $S$ of eigenvalue $\lambda<0$, it follows that the Liouville entropy has positive second derivative in the direction of $S$. Now recall that such an $S$ would generate an irreducible representation of $S O(1,3)$ with minimal $K$-type given by the $K$-orbit of $S$, i.e. with minimal $K$-type $\tau_{2}$ (here we have denoted by $\tau_{2}$ the representation of $K \approx S O(3)$ on the space of traceless symmetric tensors of rank 2). The unitary irreducible representations of $S O(1,3)$ with minimal $K$-type $\tau_{2}$ belong to the unitary principal series of $S O(1,3)$ and determined up to unitary equivalence by the value of the Casimir operator on them. From [Tay86] we obtain that the values of the eigenvalues of the Casimir operator, with our normalization, are given by $\mu^{2}-3, \mu \in \mathbb{R}$. Thus, since the Plancherel measure has support on all the interval $[-3, \infty)$, by Theorem 5.4 of [DW79], we find a cocompact lattice $\Gamma$ in $S 0(1,3)$, with spectrum of the Casimir on $\operatorname{Teich}^{\infty}(g)$ in $(-3,0)$. Then $\Gamma \backslash S O(1,3) / S O(3)$ provides us with the desired counterexample.

\section{References.}

[Ano67] D. Anosov, Geodesic flows on closed riemannian manifolds with negative sectional curvature, Proc. Steklov Inst. Math., vol. 90, American Math. Soc., Providence, 1967, English translation.

[BCG] G. Besson, G. Courtois, and S. Gallot, Les variétés hyperboliques sont des minima locaux de l' entropie topologique, Invent. Math., to appear.

[BCG94a] G. Besson, G. Courtois, and S. Gallot, Les variétés hyperboliques sont des minima de l' entropie topologique, preliminary version, 1994.

[BCG94b] G. Besson, G. Courtois, and S. Gallot, oral communication, 1994.

[Bes87] A. L. Besse, Einstein manifolds, Springer-Verlag, Berlin, Heidelberg, New York, 1987. 
[BK85] K. Burns and A. Katok, Manifolds with non-positive curvature, Ergod. Th. \& Dynam. Sys. 5 (1985), 307-317.

[Bow72a] R. Bowen, Periodic orbits for hyperbolic flows, Amer. J. Math. 94 (1972), 1-30.

[Bow72b] R. Bowen, Symbolic dynamics for hyperbolic flows, Amer. J. Math. 95 (1972), 429-459.

[Bow74] R. Bowen, Some systems with unique equilibrium states, Math. Syst. Theory 8 (1974), 193-202.

[Bow75] R. Bowen, Equilibrium states and the ergodic theory for Anosov diffeomorphisms, Lecture Notes Math., vol. 470, Springer-Verlag, BerlinHeidelberg-New York, 1975.

[BR75] R. Bowen and D. Ruelle, The ergodic theory of axiom- $A$ flows, Inv. Math. 29 (1975), 189-202.

[BW72] R. Bowen and P. Walters, Expansive one-parameter flows, J. Diff. Equs. 12 (1972), 180-193.

[Con92] G. Contreras, Regularity of topological entropy of hyperbolic flows, Math. Z. 210 (1992), 97-111.

[dlLMM86] R. de la Llave, J. M. Marco, and R. Moriyon, Canonical perturbation theory of Anosov systems and regularity results for livsic cohomology equation, Ann. Math. 123 (1986), 537-611.

[DW79] D. L. DeGeorge and N. R. Wallach, Limit formulas for multiplicities in $l^{2}(\gamma \backslash g)$ ii. the tempered spectrum, Annals of Mathematics 109 (1979), 477-495.

[FF93] A. Fathi and L. Flaminio, Infinitesimal conjugacies and WeilPetersson metric, Ann. Inst. Fourier 43,1 (1993), 279-299.

[GC50] I. M. Gel'fand and M. L. Cetlin, Finite-dimensional representations of the group of orthogonal matrices, Dokl. Akad. Nauk SSSR 71 (1950), 1017-1020, Translation: MR 11, 639.

[GK79] V. Guillemin and D. Kazhdan, Some inverse spectral results for negatively curved 2-manifolds, Topology 19 (1979), 301-312.

[Gro] M. Gromov, Three remarks on the geodesic flow, preprint.

[Gro83] M. Gromov, Filling riemannian manifolds, Jour. Diff. Geom. 18 (1983), 1-47. 
[Kat82] A. Katok, Entropy and closed geodesics, Ergod. Th. \& Dyn. Sys. 2 (1982), 339-367.

[KKPW89] A. Katok, G. Knieper, M. Pollicott, and H. Weiss, Differentiability and analyticity of topological entropy for Anosov and geodesic flows, Invent. Math. 129 (1989), 581-597.

[KKW91] A. Katok, G. Knieper, and H. Weiss, Formulas for the derivative and critical points of topological entropy for Anosov and geodesic flows, Comm. Math. Phys. 138 (1991), 19-31.

[Liv71] A. N. Livšic, Some homology properties of U-systems, Mat. Zametki 10 (1971), 555-564.

[Man79] A. Manning, Topological entropy for geodesic flows, Ann. Math. 110 (1979), 567-573.

[Mar70] G. A. Margulis, On some measure connected with U-flows, Funkt. Anal i ego Pril. 4 (1970), 62-76.

[Mor24] M. Morse, A fundamental class of geodesics on any closed surface of genus greater than one, Trans. Amer. Math. Soc. 26 (1924), 25-60.

[Pet83] K. Petersen, Ergodic theory, Cambridge University Press, Cambridge, New York, Port Chester, Melbourne, Sydney, 1983.

[Pol94] M. Pollicott, Derivatives of topological entropy for Anosov and geodesic flows, Journ. Diff. Geom. 39 (1994), 457-490.

[Rat69] M. Ratner, Invariant measure with respect to an anosov flow on a three-dimensional manifold, Sov. Math. Dokl. 10 (1969), 586-588.

[Rat73a] M. Ratner, The central limit theorem for geodesic flows on $n$ dimensional manifolds of negative curvature, Israel J. Math. 16 (1973), 181-197.

[Rat73b] M. Ratner, Markov partitions for anosov flows on n-dimensional manifolds, Israel J. Math. 15 (1973), 92-114.

[Rue78] D. Ruelle, Thermodynamic formalism: the mathematical structures of classical equilibrium statistical mechanics, Encyclopedia of mathematics and its applications, vol. 5, Addison-Wesley Pub. Co., Reading, Mass., 1978.

[Tay86] M. Taylor, Noncommutative harmonic analysis, Math. Surveys and Monographs, vol. 22, American Mathematical Soc., Providence, 1986.

[War72] G. Warner, Harmonic analysis on semi-simple lie groups, SpringerVerlag, Berlin, Heidelberg, New York, 1972. 
[Žel73] D. P. Želobenko, Compact lie groups and their representations, Translations of Mathematical Monographs, vol. 40, American Mathematical Society, Providence, 1973.

ReCEIVED JANUARY 31St, 1995.

UNIVERSITÉ DES SCIENCES ET TECHNOLOGIE DE LILLE U.F.R. DE MATHÉMATIQUES

U.R.A. 751

59655 Villeneuve D'AscQ CEDEX, FRANCE

E-MAIL ADDRESS: FLAMINIO@GAT.UNIV-LILLE1.FR 